\title{
STABILITY AND ROBUSTNESS ANALYSIS FOR SWITCHED SYSTEMS WITH TIME-VARYING DELAYS*
}

\author{
FRÉDÉRIC MAZENC ${ }^{\dagger}$, MICHAEL MALISOFF ${ }^{\ddagger}$, AND HITAY ÖZBAY§
}

\begin{abstract}
A new technique is presented for the stability and robustness analysis of nonlinear switched time-varying systems with uncertainties and time-varying delays. The delays are allowed to be discontinuous (but are required to be piecewise continuous) and arbitrarily long with known upper bounds. The technique uses an adaptation of Halanay's inequality and a trajectory-based technique, and is used for designing switched controllers to stabilize linear time-varying systems with time-varying delays.
\end{abstract}

Key words. switched system, time delay, time-varying system, asymptotic stability

AMS subject classifications. 93D21, 93C30

DOI. $10.1137 / 16 \mathrm{M} 1104895$

1. Introduction. Switched systems in continuous time have discrete switching events that use a switching signal that indicates which subsystem operates at each instant; see, e.g., [23] and [41]. They are encountered in many applications, including communication networks; see [7, 22, 39, 44, 48]. In addition, delays are frequently present in models describing engineering processes. The delays can be time varying and discontinuous, especially for control over a network, where congestion and failures in links of the network can lead to sudden changes in the routing, causing the return trip time to change abruptly. Here and in the following, we use "discontinuous delays" to mean delays that are functions of time and that are not required to be continuous, but which we will require to be piecewise continuous. See [34] for a discussion on technical problems that arise under state dependent delays, but [34] does not address switched systems.

Even for systems without switches, stability analysis for systems with timevarying delays is difficult in general [1], especially when the delay is discontinuous. Under discontinuous delays, a strategy for stability analysis consists of representing the systems as switched systems, and then applying switched systems methods. These facts motivated the recent works [41] and [46], where switched nonlinear systems with lumped delays are studied, and many other contributions, such as [6] and [40]. See also [45], for stability results for nondelayed switched systems, assuming the existence of a stable convex combination of their subsystems.

Here we study switched systems whose switching signal only depends on time. Our main result is in section 2; it is a new result that is based on combining Halanay's

\footnotetext{
* Received by the editors November 23, 2016; accepted for publication (in revised form) October 11, 2017; published electronically January 11, 2018.

http://www.siam.org/journals/sicon/56-1/M110489.html

Funding: The first and third authors were supported by PHC Bosphore 2016 France-Turkey under project 35634QM (France) and EEEAG-115E820 (TÜBİTAK-the Scientific and Technological Research Council of Turkey). The second author was supported by US NSF Grants 1408295 and 1436774 .

†Inria EPI DISCO, L2S-CNRS-CentraleSupélec, 3 rue Joliot Curie, 91192, Gif-sur-Yvette, France (frederic.mazenc@12s.centralesupelec.fr).

${ }^{\ddagger}$ Department of Mathematics, Louisiana State University, Baton Rouge, LA 70803-4918 (malisoff@lsu.edu).

$\S$ Department of Electrical and Electronics Engineering, Bilkent University, Ankara 06800, Turkey (hitay@bilkent.edu.tr).
} 
inequality [15] with the main result of [28]. See Appendix A below for a discussion on our required variant of Halanay's inequality. A key advantage of the technique is that it applies to broad classes of systems, including time-varying systems with switchings and discontinuous delays. This contrasts with much of the literature, where the timevarying delays are assumed to be continuously differentiable or the discontinuous part of the delay is assumed to be small in a suitable sense [10, 29, 43]. Our key assumptions here are a limitation on the number of switchings on all intervals of a certain length, and that the delays are bounded from above, but a key feature is that we allow the (known) upper bounds for the delays to be arbitrarily large. Hence, our work contrasts with other works that involve delays and switching such as [47] (which constructs a switching rule that yields stability properties, instead of proving stability properties for large classes of switching rules as we do here) and [41], which introduces upper bounds on the delays that make it possible to use Lyapunov-Krasovskii functionals (LKFs) to prove stability properties.

We do not assume that all of the subsystems of the switched system are stable, nor do we assume that a common Lyapunov function for the subsystems is available. This is valuable because there are several techniques for systems with common Lyapunov functions (e.g., in $[16,27]$ ), but, in many cases, common Lyapunov functions do not exist, and time-varying delays preclude applying standard invariance principles. The existence of a common strict Lyapunov function usually implies stability for any switching signal, and we aim to establish stability results under restricted switching signals because only this type of result makes it possible to cover time-varying systems with time-varying delays; see Remark 2.1 below. In section 3, we apply our main nonlinear result from section 2 to a crucial family of linear time-varying systems with time-varying delays. We show that their stability can be analyzed by finding LKFs for time-invariant systems with constant delays. We present illustrative examples in section 4, and we summarize the benefits of our work in section 5. A summary of some of this work appears in the conference paper [33], but this version adds substantial value relative to [33] by including (i) an input-to-state stability analysis for nonlinear perturbed delayed switched systems, including construction of comparison functions in the final input-to-state stability estimate, (ii) complete proofs, and (iii) a construction of a switching signal that can reduce the number of required switches for our application to linear time-varying systems; features (i)-(iii) were not present in [33], which was confined to systems that do not contain uncertainties and which only sketched its proofs.

\section{Fundamental result for nonlinear switched systems.}

2.1. Notation. We use the following notation and conventions. We omit arguments of functions when the arguments are clear from the context. Let $\mathbb{N}=\{1,2, \ldots\}$ and $\mathbb{Z}_{>0}=\mathbb{N} \cup\{0\}$. For any $k$ and $n$ in $\mathbb{N}$, the $k \times n$ matrix all of whose entries are 0 will also be denoted by 0 . The usual Euclidean norm of vectors, and the induced norm of matrices, of any dimensions are denoted by $|\cdot|$, and $I$ is the identity matrix in the dimension under consideration. The floor function is defined by $\operatorname{Floor}(x)=k$ when $k \in \mathbb{Z}_{\geq 0}$ is such that $x \in[k, k+1)$, and the ceiling function is defined by Ceiling $(r)=\min \left\{\ell \in \mathbb{Z}_{\geq 0}: \ell \geq r\right\}$ for all $r \geq 0$. We let $f\left(t^{-}\right)$denote the limit from the left of functions at those points $t$ in their domains at which the left limit is defined. Given any constant $\tau_{b}>0$, we let $C_{\text {in }}$ be the set of all continuous functions $\phi:\left[-\tau_{b}, 0\right] \rightarrow \mathbb{R}^{n}$, which we call the set of all initial functions. We define $\Xi_{t} \in C_{\text {in }}$ by $\Xi_{t}(s)=\Xi(t+s)$ for all choices of $\Xi$, s, and $t$ for which the equality is defined. The notation $C_{\text {in }}^{0}$ is used for the set of all constant initial functions. Let $\mathcal{K}_{\infty}$ be 
the set of all continuous functions $g:[0,+\infty) \rightarrow[0,+\infty)$ such that $g(0)=0, g$ is strictly increasing, and $\lim _{s \rightarrow+\infty} g(s)=+\infty$. Let $\mathcal{K} \mathcal{L}$ be the set of all continuous functions $\bar{\beta}:[0,+\infty) \times[0,+\infty) \rightarrow[0,+\infty)$ such that (i) for each $t \geq 0$, the function $f(s)=\bar{\beta}(s, t)$ is of class $\mathcal{K}_{\infty}$ and (ii) for each $s \geq 0$, the function $g(t)=\bar{\beta}(s, t)$ is nonincreasing and satisfies $\lim _{t \rightarrow+\infty} g(t)=0$, and $|f|_{\mathcal{I}}$ denotes the supremum of any real valued piecewise continuous function $f$ on any interval $\mathcal{I}$ in its domain, which can be applied to functions $f \in C_{\mathrm{in}}$. We assume that the initial times for our solutions are $t_{0}=0$, but analogous results can be shown for any initial times $t_{0} \geq 0$. By piecewise continuity of a function $f$ that is defined on $[0,+\infty)$, we mean that there is a strictly increasing unbounded sequence $s_{i}$ indexed by $i \in \mathbb{Z}_{\geq 0}$ such that $s_{0}=0$ and $f$ is continuous on $\left[s_{i}, s_{i+1}\right)$ for all $i$, where we assume that $f$ has finite left hand limits at each point in its domain.

2.2. Problem definition and preliminary remarks. We consider nonlinear time-varying switched systems with piecewise continuous delays $\tau:[0,+\infty) \rightarrow\left[0, \tau_{b}\right]$,

$$
\dot{x}(t)=f_{\sigma(t)}(t, x(t), x(t-\tau(t)), \delta(t)),
$$

where $\tau_{b}$ is a known delay bound, $x$ is valued in $\mathbb{R}^{n}, \sigma:[0,+\infty) \rightarrow\{1, \ldots, k\}$ is called the switching signal, $k \in \mathbb{N}$ and $n \in \mathbb{N}$ are arbitrary, $f_{i}$ is locally Lipschitz with respect to its second and third arguments and piecewise continuous with respect to its other arguments for all $i \in\{1, \ldots, k\}$, and the unknown piecewise continuous bounded function $\delta:[0,+\infty) \rightarrow \mathbb{R}^{d}$ models uncertainty (with $d \in \mathbb{N}$ also arbitrary). We assume that the initial functions $\phi$ are in $C_{\text {in }}$. We confine our analysis to assuming that we have time-varying piecewise continuous delays $\tau:[0,+\infty) \rightarrow[0,+\infty)$.

Let $\left\{t_{i}\right\}$ be the switching sequence for $\sigma$, i.e., the times $t_{i} \geq 0$ where $\sigma$ changes to a new value, with $t_{0}=0$ and $\sigma(t)=\sigma\left(t_{i}\right)$ for all $t \in\left[t_{i}, t_{i+1}\right)$ and $i$. We assume that there are constants $\mathcal{T}_{1}$ and $\mathcal{T}_{2}$ such that

$$
0<\mathcal{T}_{1}<t_{i+1}-t_{i} \leq \mathcal{T}_{2} \text { for all } i \in \mathbb{Z}_{\geq 0} .
$$

The constant $\mathcal{T}_{1}$ is the (minimal) dwell time, and the sequence $\left\{t_{i}\right\}$ is called a partition of $[0,+\infty)$. See [37], and see [21] for design methods that minimize dwell times to ensure stability of delay systems. These papers develop stability results under the assumption that $\inf \left\{t_{i+1}-t_{i}: i \geq 0\right\}>0$ and other assumptions (usually requiring all subsystems to be stable) [6]. In recent years, there has been considerable effort in trying to find the smallest allowable dwell times for a given switched system [21]; see Remark 2.3 for further background. We next state our technical assumptions; see the end of this subsection for their motivations.

Assumption 1 . There are $k$ locally absolutely continuous functionals denoted by $V_{j}:[0,+\infty) \times C_{\text {in }} \rightarrow[0,+\infty)$ for $j=1,2, \ldots, k$, real numbers $\alpha_{1}, \ldots, \alpha_{k}$, nonnegative constants $\beta_{1}, \ldots, \beta_{k}$, a continuous function $W: \mathbb{R}^{n} \rightarrow[0,+\infty)$, class $\mathcal{K}_{\infty}$ functions $\chi_{1}$ and $\chi_{2}$, and class $\mathcal{K}_{\infty}$ functions $\Gamma_{1}, \ldots, \Gamma_{k}$ such that

$$
\chi_{1}(|\phi(0)|) \leq V_{j}(t, \phi) \leq \chi_{2}\left(|\phi|_{\infty}\right)
$$

holds for all $\phi \in C_{\mathrm{in}}, t \in[0,+\infty)$, and $j \in\{1,2, \ldots, k\}$ and such that

$$
\dot{V}_{\sigma\left(t_{i}\right)}(t) \leq \alpha_{\sigma\left(t_{i}\right)} V_{\sigma\left(t_{i}\right)}\left(t, x_{t}\right)+\beta_{\sigma\left(t_{i}\right)} \sup _{\ell \in\left[t-\tau_{b}, t\right]} W(x(\ell))+\Gamma_{\sigma\left(t_{i}\right)}(|\delta(t)|)
$$

holds almost everywhere along all trajectories of

$$
\dot{x}(t)=f_{\sigma\left(t_{i}\right)}(t, x(t), x(t-\tau(t)), \delta(t))
$$


for almost all $t \in\left[t_{i}, t_{i+1}\right)$, all $i \in \mathbb{Z}_{\geq 0}$, and all choices of the unknown function $\delta:[0,+\infty) \rightarrow \mathbb{R}^{d}$.

Notice for later use that the preceding assumption implies that $V_{j}\left(t, x_{t}\right)$ is an absolutely continuous function of $t$ for each $j$. Notice that some of the $\alpha_{i}$ 's can be nonnegative and that no common Lyapunov function is needed; see Remark 2.1 for related discussions.

Assumption 2. The functions $V_{j}$ and $W$ from Assumption 1 admit a constant $\mu>1$ such that

$$
W(\phi(0)) \leq V_{1}(t, \phi)
$$

and

$$
V_{i}(t, \phi) \leq \mu V_{j}(t, \phi)
$$

hold for all $\phi \in C_{\text {in }}$, all $i$ and $j$ in $\{1, \ldots, k\}$, and all $t \in[0,+\infty)$.

Assumption 3. There are constants $T \geq \tau_{b}+\mathcal{T}_{2}$ and $\lambda(T)>0$ such that the inequality

$$
\int_{t-T}^{t} \alpha_{\sigma(\ell)} \mathrm{d} \ell \leq-\lambda(T)
$$

holds for all $t \geq T$.

With the above notation, we can now find a constant $\nu \geq 0$ such that

$$
\int_{t-T}^{t} \beta_{\sigma(s)} e^{\int_{s}^{t} \alpha_{\sigma(s)} \mathrm{d} \ell} \mathrm{d} s \leq \nu
$$

holds for all $t \geq T$. Note that $\nu$ exists because $\alpha_{\sigma(s)}$ and $\beta_{\sigma(s)}$ take only finitely many possible values. Let $N(r, t)$ denote the number of switching instants $t_{i}$ on $[t-r, t)$ for all $r \in(0, t]$ and $t \geq 0$, and define

$$
L(r)=\sup _{t \geq 0} N(r, t)
$$

(which is finite, by (2)). Our final assumption is the following.

Assumption 4. The inequality

$$
\mu^{L(T)+1} e^{-\lambda(T)}+\left[\frac{\mu^{L(T)+2}-1}{\mu-1}-L(T)-1\right] \nu \mu<1
$$

is satisfied.

The following remarks summarize notable features of the preceding assumptions.

Remark 2.1. Each $\alpha_{i}$ in Assumption 1 can be positive or negative, so some systems $\dot{x}(t)=f_{i}(t, x(t), x(t-\tau(t)), 0)$ for some $i$ 's may be unstable. In this case, to have global asymptotic stability for (1) when $\delta=0$, the instability of the unstable subsystems $\dot{x}(t)=f_{i}(t, x(t), x(t-\tau(t)), 0)$ should be compensated in a certain sense by the stability of other subsystems, and times $\ell \in[t-T, t]$ when $\alpha_{\sigma(\ell)}$ takes positive values must be in some sense compensated by other times $\ell \in[t-T, t]$ when $\alpha_{\sigma(\ell)}$ takes negative values in order for (7) to hold for all $t \geq T$ (as we illustrate in our examples below), which is why we cannot extend our result to switchings without 
restriction. Then (9) will also be satisfied when $\lambda(T)>0$ is large enough and $\nu$ is small enough, which will hold when Assumption 1 is satisfied for small enough constant $\beta_{i}$ 's. Assumption 1 does not make it possible to apply Razumikhin's theorem or its extensions in [30] and [49] or Halanay's inequality to prove global asymptotic stability, because several Lyapunov functions are involved.

Remark 2.2. When a common Lyapunov functional is available, i.e., $V_{1}=V_{j}$ for all $j \in\{2, \ldots, k\}$ and $\delta=0$, the problem solved below can be solved using [30] and [49]. Recall that global uniform exponential stability of a switched system does not imply that its subsystems have a common Lyapunov function; see [23, section 2.1.5]. Also, if a switched system is input-to-state stable (ISS) under arbitrary switching, then uniform (with respect to the switching signals) ISS is equivalent to the existence of a common ISS Lyapunov function; see [26]. Using [32, Lemma A.1], we can extend our result to cases where the $\alpha_{i}$ 's and $\beta_{i}$ 's are time varying. For simplicity, we do not present this extension. Setting

$$
U(t, \phi)=V_{\sigma(t)}(t, \phi),
$$

Assumption 1 gives

$$
\dot{U}(t) \leq a(t) U\left(t, x_{t}\right)+b(t) \sup _{\ell \in\left[t-\tau_{b}, t\right]} W(x(\ell))+\max \left\{\Gamma_{i}(|\delta(t)|): 1 \leq i \leq k\right\}
$$

almost everywhere, where $a(t)=\alpha_{\sigma(t)}$ and $b(t)=\beta_{\sigma(t)}$. Inequality (10) does not make it possible to conclude as in [30], because $U$ is discontinuous. The proof of our main result will show that if the $\chi_{i}$ 's from Assumption 1 are quadratic functions, then $x(t)$ converges exponentially to zero when $\delta=0$. The inequalities (6) in Assumption 2 are a standard assumption; see [23]. The fact that we do not assume periodicity of the systems in time also puts our switched delay systems results outside the scope of existing results, e.g., since the Floquet approach for eliminating the time dependencies would not apply without periodicity.

Remark 2.3. The constant $\mathcal{T}_{2}$ in (2) is the maximal dwell time; see, e.g., significant works such as $[3,4,5]$ on linear systems. In the average dwell time case, our use of $\mathcal{T}_{2}$ is also called the reverse dwell time condition; see, e.g., the work [17] that uses common Lyapunov functions. See also the foundational literature [12, 18, 23, 35] for average and minimal dwell times. Also, conditions of the form (2) are called ranged dwell time conditions and can be relaxed in the presence of (mode-dependent) ranged dwell time conditions; see [3] and its references. See also [9, 34] which study stability under minimal and average dwell times in the case of switching between a family of asymptotically stable systems; and see $[8,12,42]$ for other notable works related to finding smallest dwell times, which are not about delay systems. Other significant works that include both delays and switches include the works [24, 25], which employ a common Lyapunov functional approach that we do not require here, since as we noted in the introduction, one of our objectives in this work is to cover cases where the common Lyapunov functionals may not be available. Therefore, while works such as $[24,25]$ can be applied when all of our $\alpha_{i}$ 's are negative and a common Lyapunov functional is known, our ability to cover much more general cases where some of the subsystems can be unstable using our novel Halanay and trajectory-based approaches makes our work significantly novel and valuable.

2.3. Main result. With the notation introduced above, and by letting $\rho \in(0,1)$ denote the constant left side of (9), we next state our main result, in which the final 
estimate is a special case of ISS [20] (which includes global asymptotic stability of (1) to the origin in the special case where the perturbation $\delta$ is the zero function). The result is novel and significant, even in the special case where $\delta$ is 0 , due to its use of the contractiveness condition in (9) and its allowing discontinuous arbitrarily large $\tau$ 's and nonlinear systems.

TheOREm 2.4. Let the system (1) satisfy Assumptions 1-4. Then the functions and the positive constants

$$
\begin{aligned}
\bar{\beta}(s, \ell) & =\chi_{1}^{-1}\left(2 \max \left\{2 e^{\frac{\ln (\rho)}{T+\tau_{b}}\left(\ell-T-\tau_{b}\right)}, e^{T+\tau_{b}-\ell}\right\} e^{R_{*} a_{*} \mathcal{T}_{2}} \chi_{2}(s)\right), \\
\bar{\gamma}(r) & =2 \chi_{1}^{-1}\left(2 \max \left\{B_{*}, 2 a_{*} \mathcal{T}_{2} \sum_{j=1}^{R_{*}} e^{j a_{*} \mathcal{T}_{2}}\right\} \max \left\{\Gamma_{i}(r): 1 \leq i \leq r\right\}\right), \\
R_{*} & =\operatorname{Ceiling}\left(\frac{T+\tau_{b}}{\mathcal{T}_{1}}\right)+1, \\
a_{*} & =\max \left\{1, \max \left\{\alpha_{i}+\mu \beta_{i}: 1 \leq i \leq k\right\}\right\}, \text { and } B_{*}=\frac{\mu^{L(T)+1}}{(1-\rho)^{2}} T e^{\max _{i}\left|\alpha_{i}\right| T}
\end{aligned}
$$

are such that $\bar{\beta} \in \mathcal{K} \mathcal{L}, \bar{\gamma} \in \mathcal{K}_{\infty}$, and

$$
|x(t)| \leq \bar{\beta}\left(|x|_{\left[-\tau_{b}, 0\right]}, t\right)+\bar{\gamma}\left(|\delta|_{[0, t]}\right)
$$

holds for all $t \geq 0$ along all solutions of (1) for all choices of the piecewise continuous bounded function $\delta:[0,+\infty) \rightarrow \mathbb{R}^{d}$.

Proof. For the sake of brevity, we use the notation

$$
\begin{gathered}
a(t)=\alpha_{\sigma(t)}, b(t)=\beta_{\sigma(t)}, \bar{\Gamma}(r)=\max \left\{\Gamma_{i}(r): 1 \leq i \leq k\right\}, \\
\text { and } U(t, \phi)=V_{\sigma(t)}(t, \phi)
\end{gathered}
$$

for all $t \geq 0, r \geq 0$, and $\phi \in C_{\mathrm{in}}$. We can use Assumptions 1-2 to prove that the system $(1)$ is forward complete. In fact, forward completeness on $\left[0, t_{1}\right]$ follows by combining Assumption 1 with the upper bounds $W(x(\ell)) \leq \mu V_{j}(\ell, x(\ell))$ from Assumption 2, and applying Gronwall's inequality to the function

$$
\mathcal{F}(t)=\sup \left\{V_{j}(\ell, x(\ell)): \ell \in[0, t], 1 \leq j \leq k\right\}
$$

for all $t \in\left[0, t_{1}\right]$, and then arguing inductively.

We next analyze the behavior of $U$ along the trajectories of (1) for a fixed choice of $\delta$. Observe that Assumption 1 implies that for each $t \geq 0$, and for the unique choices of $i \in \mathbb{Z}_{\geq 0}$ and $r \in\{1,2, \ldots, k\}$ (depending on $t$ ) such that $t \in\left[t_{i}, t_{i+1}\right.$ ) and $\sigma\left(t_{i}\right)=r$, and for any $t_{*} \in\left[t_{i}, t\right]$, the inequality

$$
\begin{aligned}
V_{r}\left(t, x_{t}\right) \leq & e^{\alpha_{r}\left(t-t_{*}\right)} V_{r}\left(t_{*}, x_{t_{*}}\right) \\
& +\int_{t_{*}}^{t} e^{\alpha_{r}(t-s)}\left(\beta_{r} \sup _{\ell \in\left[s-\tau_{b}, s\right]} W(x(\ell))+\Gamma_{r}(|\delta(s)|)\right) \mathrm{d} s
\end{aligned}
$$

is satisfied. This can be shown by first noting that for all $p \in\left[t_{i}, t_{i+1}\right)$, condition (4) from Assumption 1 gives

$$
\dot{V}_{r}(p) \leq \alpha_{r} V_{r}\left(p, x_{p}\right)+\beta_{r} \sup _{\ell \in\left[p-\tau_{b}, p\right]} W(x(\ell))+\Gamma_{r}(|\delta(p)|),
$$

Copyright $@$ by SIAM. Unauthorized reproduction of this article is prohibited. 
and then we can multiply both sides of (15) by the integrating factor $e^{-\alpha_{r} p}$, then move the resulting term $e^{-\alpha_{r} p} \alpha_{r} V_{r}\left(p, x_{p}\right)$ from the right side to the left side, and then integrate both sides over the interval $\left[t_{*}, t\right]$ to get $(14)$.

It follows that for all $i \in \mathbb{Z}_{\geq 0}$, all $t \in\left[t_{i}, t_{i+1}\right)$, and all $t_{*} \in\left[t_{i}, t\right]$, the inequality

$$
\begin{aligned}
U\left(t, x_{t}\right) \leq & e^{\int_{t_{*}}^{t} a(s) \mathrm{d} s} U\left(t_{*}, x_{t_{*}}\right) \\
& +\int_{t_{*}}^{t} e^{\int_{s}^{t} a(p) \mathrm{d} p}\left(b(s) \sup _{\ell \in\left[s-\tau_{b}, s\right]} W(x(\ell))+\Gamma_{\sigma(s)}(|\delta(s)|)\right) \mathrm{d} s
\end{aligned}
$$

is satisfied. We introduce the simplifying notation

$$
\mathcal{E}(\ell, t)=e^{\int_{\ell}^{t} a(s) \mathrm{d} s}, \quad \mathcal{G}_{i}=\mu \mathcal{E}\left(t_{i-1}, t_{i}\right), \text { and } w(t)=\sup _{\ell \in\left[t-\tau_{b}, t\right]} W(x(\ell)) .
$$

From (16) with the choice $t_{*}=t_{i}$, and using the simplifying notation $U(t)$ to mean $U\left(t, x_{t}\right)$, we deduce that if $i \geq 1$, then

$$
\begin{aligned}
U(t) & \leq \mathcal{E}\left(t_{i}, t\right) U\left(t_{i}\right)+b(t) \int_{t_{i}}^{t} \mathcal{E}(\ell, t) w(\ell) \mathrm{d} \ell+\int_{t_{i}}^{t} \mathcal{E}(\ell, t) \bar{\Gamma}(|\delta(\ell)|) \mathrm{d} \ell \\
& \leq \mu \mathcal{E}\left(t_{i}, t\right) U\left(t_{i}^{-}\right)+\int_{t_{i}}^{t} \mathcal{E}(\ell, t) w^{\sharp}(\ell) \mathrm{d} \ell,
\end{aligned}
$$

where $w^{\sharp}(\ell)=b(\ell) w(\ell)+\bar{\Gamma}(|\delta(\ell)|)$, and where the last inequality follows from (6) in Assumption 2.

Next, let $t \geq T, q \geq 0$, and $j \in \mathbb{Z}_{\geq 0}$ be such that $t \in\left[t_{j}, t_{j+1}\right), j-1 \geq q$, and $t-T \in\left[t_{j-q-1}, t_{j-q}\right)$. We first consider the case where $q \geq 2$; later in the proof, we explain the changes needed to cover cases where $q \in\{0,1\}$. From the first inequality in (18) (with the choice $i=l-1$, and taking the left limit $t \rightarrow t_{l}^{-}$in (18)), we deduce that for all integers $l \geq 1$, the inequality

$$
U\left(t_{l}^{-}\right) \leq \mathcal{E}\left(t_{l-1}, t_{l}\right) U\left(t_{l-1}\right)+\int_{t_{l-1}}^{t_{l}} \mathcal{E}\left(\ell, t_{l}\right) w^{\sharp}(\ell) \mathrm{d} \ell
$$

is satisfied. Consequently, using (6) in Assumption 2 and the nonnegativity of the $\mathcal{G}_{j}$ 's, we obtain

$$
\begin{gathered}
U\left(t_{j}^{-}\right) \leq \mathcal{G}_{j} U\left(t_{j-1}^{-}\right)+\int_{t_{j-1}}^{t_{j}} \mathcal{E}\left(\ell, t_{j}\right) w^{\sharp}(\ell) \mathrm{d} \ell, \\
\mathcal{G}_{j} U\left(t_{j-1}^{-}\right) \leq \mathcal{G}_{j} \mathcal{G}_{j-1} U\left(t_{j-2}^{-}\right)+\mathcal{G}_{j} \int_{t_{j-2}}^{t_{j-1}} \mathcal{E}\left(\ell, t_{j-1}\right) w^{\sharp}(\ell) \mathrm{d} \ell,
\end{gathered}
$$

$$
\begin{aligned}
\prod_{r=j-q+2}^{j} \mathcal{G}_{r} U\left(t_{j-q+1}^{-}\right) \leq & \prod_{r=j-q+1}^{j} \mathcal{G}_{r} U\left(t_{j-q}^{-}\right) \\
& +\prod_{r=j-q+2}^{j} \mathcal{G}_{r} \int_{t_{j-q}}^{t_{j-q+1}} \mathcal{E}\left(\ell, t_{j-q+1}\right) w^{\sharp}(\ell) \mathrm{d} \ell,
\end{aligned}
$$

where we used the fact that $q \geq 2$. 
By moving the left side terms in the last $q-1$ inequalities in (20) to the corresponding right sides of the last $q-1$ inequalities in (20), and then summing the inequalities in (20), we obtain

$$
\begin{aligned}
U\left(t_{j}^{-}\right) \leq & \prod_{r=j-q+1}^{j} \mathcal{G}_{r} U\left(t_{j-q}^{-}\right)+\int_{t_{j-1}}^{t_{j}} \mathcal{E}\left(\ell, t_{j}\right) w^{\sharp}(\ell) \mathrm{d} \ell \\
& +\mathcal{G}_{j} \int_{t_{j-2}}^{t_{j-1}} \mathcal{E}\left(\ell, t_{j-1}\right) w^{\sharp}(\ell) \mathrm{d} \ell \\
& +\cdots+\prod_{r=j-q+2}^{j} \mathcal{G}_{r} \int_{t_{j-q}}^{t_{j-q+1}} \mathcal{E}\left(\ell, t_{j-q+1}\right) w^{\sharp}(\ell) \mathrm{d} \ell .
\end{aligned}
$$

Also, using (16) with the choice $t_{*}=t-T$ (and by letting $t \rightarrow t_{j-q}^{-}$in (16)), we obtain

$$
U\left(t_{j-q}^{-}\right) \leq \mathcal{E}\left(t-T, t_{j-q}\right) U(t-T)+\int_{t-T}^{t_{j-q}} \mathcal{E}\left(\ell, t_{j-q}\right) w^{\sharp}(\ell) \mathrm{d} \ell,
$$

since $t-T \in\left[t_{j-q-1}, t_{j-q}\right)$.

Next note that $\mathcal{G}_{h} \mathcal{G}_{h+1} \cdots \mathcal{G}_{j}=\mu^{j-h+1} \mathcal{E}\left(t_{h-1}, t_{j}\right)$ if $j \geq h$. Hence, if $q \geq 2$, then (21) gives

$$
\begin{aligned}
U\left(t_{j}^{-}\right) \leq & \mu^{q} \mathcal{E}\left(t_{j-q}, t_{j}\right) U\left(t_{j-q}^{-}\right)+\int_{t_{j-1}}^{t_{j}} \mathcal{E}\left(\ell, t_{j}\right) w^{\sharp}(\ell) \mathrm{d} \ell \\
& +\left\{\mu \int_{t_{j-2}}^{t_{j-1}} \mathcal{E}\left(\ell, t_{j}\right) w^{\sharp}(\ell) \mathrm{d} \ell+\cdots+\mu^{q-1} \int_{t_{j-q}}^{t_{j-q+1}} \mathcal{E}\left(\ell, t_{j}\right) w^{\sharp}(\ell) \mathrm{d} \ell\right\} .
\end{aligned}
$$

Using (22) to upper bound the $U\left(t_{j-q}^{-}\right)$in (23), we conclude that if $q \geq 2$, then

$$
\begin{aligned}
U\left(t_{j}^{-}\right) \leq & \mu^{q} \mathcal{E}\left(t_{j-q}, t_{j}\right) \mathcal{E}\left(t-T, t_{j-q}\right) U(t-T) \\
& +\mu^{q} \mathcal{E}\left(t_{j-q}, t_{j}\right) \int_{t-T}^{t_{j-q}} \mathcal{E}\left(\ell, t_{j-q}\right) w^{\sharp}(\ell) \mathrm{d} \ell+\int_{t_{j-1}}^{t_{j}} \mathcal{E}\left(\ell, t_{j}\right) w^{\sharp}(\ell) \mathrm{d} \ell \\
& +\left\{\mu \int_{t_{j-2}}^{t_{j-1}} \mathcal{E}\left(\ell, t_{j}\right) w^{\sharp}(\ell) \mathrm{d} \ell+\cdots+\mu^{q-1} \int_{t_{j-q}}^{t_{j-q+1}} \mathcal{E}\left(\ell, t_{j}\right) w^{\sharp}(\ell) \mathrm{d} \ell\right\} \\
= & \mu^{q} \mathcal{E}\left(t-T, t_{j}\right) U(t-T)+\int_{t_{j-1}}^{t_{j}} \mathcal{E}\left(\ell, t_{j}\right) w^{\sharp}(\ell) \mathrm{d} \ell \\
& +\left\{\mu \int_{t_{j-2}}^{t_{j-1}} \mathcal{E}\left(\ell, t_{j}\right) w^{\sharp}(\ell) \mathrm{d} \ell+\cdots+\mu^{q-1} \int_{t_{j-q}}^{t_{j-q+1}} \mathcal{E}\left(\ell, t_{j}\right) w^{\sharp}(\ell) \mathrm{d} \ell\right\} \\
& +\mu^{q} \int_{t_{-T}}^{t_{j-q}} \mathcal{E}\left(\ell, t_{j}\right) w^{\sharp}(\ell) \mathrm{d} \ell,
\end{aligned}
$$

Copyright $@$ by SIAM. Unauthorized reproduction of this article is prohibited. 
which we can use to upper bound $U\left(t_{j}^{-}\right)$from (18) (with $i=j$ ) to get

$$
\begin{aligned}
U(t) \leq & \mu^{q+1} \mathcal{E}(t-T, t) U(t-T)+\int_{t_{j}}^{t} \mathcal{E}(\ell, t) w^{\sharp}(\ell) \mathrm{d} \ell+\mu \int_{t_{j-1}}^{t_{j}} \mathcal{E}(\ell, t) w^{\sharp}(\ell) \mathrm{d} \ell \\
& +\left\{\mu^{2} \int_{t_{j-2}}^{t_{j-1}} \mathcal{E}(\ell, t) w^{\sharp}(\ell) \mathrm{d} \ell+\cdots+\mu^{q} \int_{t_{j-q}}^{t_{j-q+1}} \mathcal{E}(\ell, t) w^{\sharp}(\ell) \mathrm{d} \ell\right\} \\
& +\mu^{q+1} \int_{t-T}^{t_{j-q}} \mathcal{E}(\ell, t) w^{\sharp}(\ell) \mathrm{d} \ell \\
\leq & \mu^{q+1} e^{-\lambda(T)} U(t-T)+\Lambda(t) \sup _{\ell \in\left[t-\tau_{b}-T, t\right]} W(x(\ell))+\Lambda_{*}(t) \bar{\Gamma}\left(|\delta|_{[0, t]}\right),
\end{aligned}
$$

where

$$
\begin{aligned}
\Lambda(t)= & \int_{t_{j}}^{t} b(\ell) \mathcal{E}(\ell, t) \mathrm{d} \ell+\mu \int_{t_{j-1}}^{t_{j}} b(\ell) \mathcal{E}(\ell, t) \mathrm{d} \ell \\
& +\left\{\mu^{2} \int_{t_{j-2}}^{t_{j-1}} b(\ell) \mathcal{E}(\ell, t) \mathrm{d} \ell+\cdots+\mu^{q} \int_{t_{j-q}}^{t_{j-q+1}} b(\ell) \mathcal{E}(\ell, t) \mathrm{d} \ell\right\} \\
& +\mu^{q+1} \int_{t-T}^{t_{j-q}} b(\ell) \mathcal{E}(\ell, t) \mathrm{d} \ell \text { and } \Lambda_{*}(t)=\mu^{q+1} \int_{t-T}^{t} \mathcal{E}(\ell, t) \mathrm{d} \ell
\end{aligned}
$$

by (7). Since $\mu>1$ and $q \leq L(T)$, our condition (8) on $\nu$ implies that if $q \geq 2$, then

$$
\begin{aligned}
\Lambda(t)= & \int_{t-T}^{t} b(\ell) e^{\int_{\ell}^{t} a(s) \mathrm{d} s} \mathrm{~d} \ell+(\mu-1) \int_{t_{j-1}}^{t_{j}} b(\ell) \mathcal{E}(\ell, t) \mathrm{d} \ell \\
& +\left\{\left(\mu^{2}-1\right) \int_{t_{j-2}}^{t_{j-1}} b(\ell) \mathcal{E}(\ell, t) \mathrm{d} \ell+\cdots+\left(\mu^{q}-1\right) \int_{t_{j-q}}^{t_{j-q+1}} b(\ell) \mathcal{E}(\ell, t) \mathrm{d} \ell\right\} \\
& +\left(\mu^{q+1}-1\right) \int_{t-T}^{t_{j-q}} b(\ell) \mathcal{E}(\ell, t) \mathrm{d} \ell \\
\leq & \int_{t-T}^{t} b(\ell) e^{\int_{\ell}^{t} a(s) \mathrm{d} s} \mathrm{~d} \ell \\
& +\left[\mu-1+\mu^{2}-1+\cdots+\mu^{L(T)+1}-1\right] \int_{t-T}^{t} b(\ell) e^{\int_{\ell}^{t} a(s) \mathrm{d} s} \mathrm{~d} \ell \\
\leq & {\left[\frac{\mu^{L(T)+2}-1}{\mu-1}-L(T)-1\right] \nu, }
\end{aligned}
$$

where the last inequality also used the formula for the geometric sum. Also, $\Lambda_{*}(t) \leq$ $\mu^{L(T)+1} T e^{\max _{i}\left|\alpha_{i}\right| T}$ for all $t \geq 0$. Using (26) to upper bound $\Lambda(t)$ in (25), recalling that $\mu>1$ and $q \leq L(T)$, and using Assumption 2 to upper bound $\sup _{\ell \in\left[t-\tau_{b}-T, t\right]} W(x(\ell))$, we conclude that if $q \geq 2$, then for all $t \geq T+\tau_{b}$, we obtain

$$
\begin{aligned}
U(t) \leq & \mu^{L(T)+1} e^{-\lambda(T)} U(t-T) \\
& +\left[\frac{\mu^{L(T)+2}-1}{\mu-1}-L(T)-1\right] \nu \mu \sup _{\ell \in\left[t-\tau_{b}-T, t\right]} U(\ell)+\bar{\Lambda} \bar{\Gamma}\left(|\delta|_{[0, t]}\right),
\end{aligned}
$$

where $\bar{\Lambda}=\mu^{L(T)+1} T e^{\max _{i}\left|\alpha_{i}\right| T}$. On the other hand, if $q=0$, then we can use (22) to upper bound $U\left(t_{j}^{-}\right)$from (18) to obtain (27) in this case as well (since the quantity

Copyright $@$ by SIAM. Unauthorized reproduction of this article is prohibited. 
in square brackets in (27) is larger than $\mu$ ). Finally, if $q=1$, then (27) again follows, by arguing as above except with the quantities in curly braces from (23) through (26) removed.

Therefore, our choice of the constant $\rho \in(0,1)$ (which was chosen to be the left side of (9)) and the choices $\bar{w}(\ell)=U\left(\ell+T+\tau_{b}\right)$ give $\bar{w}(t) \leq \rho|\bar{w}|_{\left[t-T-\tau_{b}, t\right]}+d(t)$ for all $t \geq 0$, where

$$
d(t)=\bar{\Lambda} \bar{\Gamma}\left(|\delta|_{\left[0, T+\tau_{b}+t\right]}\right) .
$$

We can therefore use [28, Lemma 1] (which we also state in Appendix A below) with the choice $T_{*}=T+\tau_{b}$ and Assumption 4 to obtain

$$
U(t) \leq e^{\frac{\ln (\rho)}{T_{*}}\left(t-T_{*}\right)}|U|_{\left[0, T_{*}\right]}+B_{*} \bar{\Gamma}\left(|\delta|_{[0, t]}\right)
$$

for all $t \geq T_{*}$. Also, Assumptions 1-2 and our choice of $a_{*}$ in (11) give

$$
\frac{d}{d t} V_{i}\left(t, x_{t}\right) \leq a_{*}\left(\sup _{\ell \in\left[t-\tau_{b}, t\right]} V_{i}\left(\ell, x_{\ell}\right)+\Gamma_{i}\left(|\delta|_{[0, t]}\right)\right)
$$

for almost all $t \in\left[t_{i}, t_{i+1}\right)$ and all $i \in \mathbb{Z}_{\geq 0}$. Integrating both sides of (29) (with $i=\sigma(0))$ over $[0, t]$ and then using Gronwall's inequality gives

$$
\mathcal{F}(t) \leq e^{a_{*} \mathcal{T}_{2}}\left(\mathcal{F}(0)+a_{*} \mathcal{T}_{2} \bar{\Gamma}\left(|\delta|_{[0, t]}\right)\right)
$$

for all $t \in\left[0, t_{1}\right)$ and therefore also

$$
\mathcal{F}(t) \leq e^{2 a_{*} \mathcal{T}_{2}} \mathcal{F}(0)+a_{*} \mathcal{T}_{2}\left(e^{2 a_{*} \mathcal{T}_{2}}+e^{a_{*} \mathcal{T}_{2}}\right) \bar{\Gamma}\left(|\delta|_{[0, t]}\right)
$$

for all $t \in\left[0, t_{2}\right)$, where

$$
\mathcal{F}(t)=\sup \left\{V_{j}(\ell, x(\ell)): \ell \in[0, t], 1 \leq j \leq k\right\} .
$$

Reasoning inductively gives

$$
\mathcal{F}(t) \leq e^{R_{*} a_{*} \mathcal{T}_{2}} \mathcal{F}(0)+a_{*} \mathcal{T}_{2}\left(\sum_{j=1}^{R_{*}} e^{j a_{*} \mathcal{T}_{2}}\right) \bar{\Gamma}\left(|\delta|_{[0, t]}\right)
$$

for all $t \in\left[0, t_{R_{*}}\right)$, and our choice of $R_{*}$ in (11) gives $t_{R_{*}} \geq \mathcal{T}_{1} R_{*}>T_{*}$. Hence, for all $t \in\left[0, T_{*}\right]$, we have

$$
\chi_{1}(|x(t)|) \leq U(t) \leq e^{R_{*} a_{*} \mathcal{T}_{2}} \chi_{2}\left(|x|_{\left[-\tau_{b}, 0\right]}\right)+a_{*} \mathcal{T}_{2}\left(\sum_{j=1}^{R_{*}} e^{j a_{*} \mathcal{T}_{2}}\right) \bar{\Gamma}\left(|\delta|_{[0, t]}\right)
$$

by Assumption 1. Using the subadditivity property $\chi_{i}(a+b) \leq \chi_{i}(2 a)+\chi_{i}(2 b)$ of our functions $\chi_{i} \in \mathcal{K}_{\infty}$ for all $a \geq 0$ and $b \geq 0$ (which follows by separately considering the cases $a \geq b$ and $a<b),(28)$ and (32) give

$$
\begin{aligned}
|x(t)| \leq & \chi_{1}^{-1}\left(2 e^{\frac{\ln (\rho)}{T_{*}}\left(t-T_{*}\right)}|U|_{\left[0, T_{*}\right]}\right)+\chi_{1}^{-1}\left(2 B_{*} \bar{\Gamma}\left(|\delta|_{[0, t]}\right)\right) \\
\leq & \chi_{1}^{-1}\left(4 e^{\frac{\ln (\rho)}{T_{*}}\left(t-T_{*}\right)} e^{R_{*} a_{*} \mathcal{T}_{2}} \chi_{2}\left(|x|_{\left[-\tau_{b}, 0\right]}\right)\right) \\
& +\chi_{1}^{-1}\left(4 a_{*} \mathcal{T}_{2}\left(\sum_{j=1}^{R_{*}} e^{j a_{*} \mathcal{T}_{2}}\right) \bar{\Gamma}\left(|\delta|_{[0, t]}\right)\right)+\chi_{1}^{-1}\left(2 B_{*} \bar{\Gamma}\left(|\delta|_{[0, t]}\right)\right)
\end{aligned}
$$

Copyright (c) by SIAM. Unauthorized reproduction of this article is prohibited. 
for all $t \geq T_{*}$ and

$$
\begin{aligned}
|x(t)| \leq & \chi_{1}^{-1}\left(2 e^{T_{*}-t} e^{R_{*} a_{*} \mathcal{T}_{2}} \chi_{2}\left(|x|_{\left[-\tau_{b}, 0\right]}\right)\right) \\
& +\chi_{1}^{-1}\left(2 a_{*} \mathcal{T}_{2}\left(\sum_{j=1}^{R_{*}} e^{j a_{*} \mathcal{T}_{2}}\right) \bar{\Gamma}\left(|\delta|_{[0, t]}\right)\right)
\end{aligned}
$$

for all $t \in\left[0, T_{*}\right]$. The theorem now follows by choosing the maximum of the two right sides in (33)-(34).

3. Application to linear time-varying systems. Theorem 2.4 is valuable because of its ability to handle arbitrarily large nonconstant delays in large classes of nonlinear switched systems. We next apply Theorem 2.4 to a fundamental family of linear time-varying systems with time-varying lumped delays. In the rest of this paper, we assume that the initial functions for our systems are constant on $(-\infty, 0]$ (but see Remark 3.6 for ways to relax this assumption). For simplicity, we do not assume that the systems in this section have switches, but we illustrate connections with switched systems. Throughout this section, we assume that the perturbations in the system are 0 (in which case Theorem 2.4 gives global asymptotic stability), but since the systems are linear, it is straightforward to extend the results to follow to allow perturbed systems. A notable feature in this section is that we do not require our time-varying linear systems to be periodic, so it is not possible to use Floquet theory to reduce the problems to simpler problems for linear time invariant systems, and in addition, the Floquet method does not in general provide an explicit transformation that can eliminate the periodic dependence.

3.1. Problem definition and preliminary remarks. In this subsection we consider the system

$$
\dot{x}(t)=A(t) x(t)+B(t) x(t-\tau(t)),
$$

where $x$ takes values in $\mathbb{R}^{n}$ for any $n \in \mathbb{N}$ and $t \geq 0$ with a constant initial function $\phi \in C_{\mathrm{in}}^{0}$. The following assumptions are in effect in this subsection.

Assumption 5. The functions $A, B$, and $\tau$ are piecewise $C^{1}$, and there are constants $a_{b}, b_{b}$, and $\tau_{b}$ such that $|A(t)| \leq a_{b},|B(t)| \leq b_{b}$, and $\tau(t) \leq \tau_{b}$ hold for all $t \geq 0$.

Assumption 6. There exist an integer $k \in \mathbb{N}$, a switching signal $\sigma:[0,+\infty) \rightarrow$ $\{1,2, \ldots, k\}$ whose switching instants $\left\{t_{i}\right\}$ admit positive constants $\mathcal{T}_{1}$ and $\mathcal{T}_{2}$ that satisfy (2), a finite set $\left\{\left(A_{i}, B_{i}, \tau_{i}\right): i \in\{1,2, \ldots, k\}\right\}$ in $\mathbb{R}^{n \times n} \times \mathbb{R}^{n \times n} \times[0,+\infty)$, and nonnegative constants $\bar{a}, \bar{b}$, and $\bar{\tau}$ such that

$$
\left|A(t)-A_{\sigma(t)}\right| \leq \bar{a}, \quad\left|B(t)-B_{\sigma(t)}\right| \leq \bar{b}, \quad \text { and } \quad\left|\tau(t)-\tau_{\sigma(t)}\right| \leq \bar{\tau}
$$

hold for all $t \geq 0$.

See Appendix D for an illustration of how such switching signals can be found to satisfy the preceding assumption.

Assumption 7. For each $i \in\{1, \ldots, k\}$, there exist a locally absolutely continuous function $V_{i}: C_{\text {in }} \rightarrow[0,+\infty)$, a real constant $\alpha_{i}$, and a positive constant $\gamma_{i}$ such that for all piecewise continuous functions $\delta_{i}:[0,+\infty) \rightarrow \mathbb{R}^{n}$, the time derivative of $V_{i}$ along all trajectories of

$$
\dot{z}(t)=A_{i} z(t)+B_{i} z\left(t-\tau_{i}\right)+\delta_{i}(t)
$$


satisfies

$$
\dot{V}_{i} \leq \alpha_{i} V_{i}\left(z_{t}\right)+\gamma_{i}\left|\delta_{i}(t)\right|^{2}
$$

for almost all $t$, where the triples $\left(A_{i}, B_{i}, \tau_{i}\right)$ are from Assumption 6. Moreover, there are constants $\Psi_{1}>0$ and $\Psi_{2}>0$ such that for all $\phi \in C_{\text {in }}$ and all $i \in\{1, \ldots, k\}$, we have

$$
\Psi_{1}|\phi(0)|^{2} \leq V_{i}(\phi) \leq \Psi_{2}|\phi|_{\left[-\tau_{b}, 0\right]}^{2} .
$$

Also, the $\alpha_{i}$ 's satisfy Assumption 3 with $\sigma$ as defined in Assumption 6. Finally, there is a constant $\mu>1$ such that $V_{i}(\phi) \leq \mu V_{j}(\phi)$ holds for all $(i, j) \in\{1, \ldots, m\}^{2}$ and for all $\phi \in C_{\text {in }}$.

Assumption 8. Assumption 4 is satisfied, where $\nu$ is a constant that satisfies (8) as before, and $\sigma, \mu$, and the $\alpha_{i}$ 's are defined as in Assumptions 6-7, and the $\beta_{i}$ 's are defined by

$$
\beta_{i}=\frac{\gamma_{i}\left[\bar{a}+\bar{b}+b_{b} \bar{\tau}\left(a_{b}+b_{b}\right)\right]^{2}}{\Psi_{1}}
$$

for $i=1,2, \ldots, k$.

Before stating our main result of this section, we point out several notable features of Assumptions 5-8.

Remark 3.1. In Assumption 6, we can always choose $\bar{a}=a_{b}+\max _{i}\left|A_{i}\right|, \bar{b}=$ $b_{b}+\max _{i}\left|B_{i}\right|$, and $\bar{\tau}=\tau_{b}+\max _{i} \tau_{i}$. However, these choices will not in general provide the smallest possible values for the left side of (9) in Assumption 4. We can view the triplets $\left(A_{i}, B_{i}, \tau_{i}\right)$ as the coefficient matrices and constant delays of linear time invariant systems; then time-varying perturbations around these systems (along with switchings) determine the linear time-varying system (35). Therefore, stability of the linear time invariant switched systems together with certain conditions on the bounds defined in Assumption 6 will guarantee stability of the linear time-varying system.

Remark 3.2. Assumption 6 covers the important special case where $A, B$, and $\tau$ all have some period $\mathcal{P}>0$ by choosing $t_{i}=i \mathcal{P} / k$ and

$$
\left(A_{i}, B_{i}, \tau_{i}\right)=(A(i \mathcal{P} / k), B(i \mathcal{P} / k), \tau(i \mathcal{P} / k))
$$

for all $i \in\{1,2, \ldots, k\}$, in which case the system (37) is

$$
\dot{z}(t)=A\left(\frac{i \mathcal{P}}{k}\right) z(t)+B\left(\frac{i \mathcal{P}}{k}\right) z\left(t-\tau\left(\frac{i \mathcal{P}}{k}\right)\right)+\delta_{i}(t),
$$

and we take the switching signal $\sigma$ defined by $\sigma(t)=i$ for all $t \in[i \mathcal{P} / k,(i+1) \mathcal{P} / k)$ and $i \in\{1, \ldots, k\}$. However, Assumption 6 is much more general, e.g., because we do not require periodicity of the coefficient matrices or periodicity of the delay, nor do we require the $t_{i}$ 's to be evenly spaced. Even when $A, B$, and $\tau$ are periodic, it is worth trying to find a switching sequence with fewer switching instants $\{i \mathcal{P} / k\}$ on each interval, since doing so can help keep $\rho$ small for given choices of $\mu$ and $\nu$, where $\rho$ is the left side of (9) as before. These comments motivate Appendix B below.

Remark 3.3. For systems (37), linear matrix inequalities [14] make it possible to construct time invariant quadratic LKFs. In Appendix C, we explain how to construct LKFs to satisfy (38)-(39). We do not require the constants $\alpha_{i}$ to be nonpositive. Thus, we can allow some of the systems (37) to be unstable.

Remark 3.4. Since we only require $\tau(t)$ to be piecewise $C^{1}$ and bounded (instead of $C^{1}$ ), sampling (and many other cases) can be handled. Indeed, sampling can be 
represented as sawtooth shaped delay functions, whose values are 0 at the sampling times [11]. The present setup allows nonuniform sampling and drifts in the sampling clocks. This further motivates our work, because controls are typically implemented using sampled observations. While hybrid systems methods $[13,19,36]$ can be specialized to sawtooth delays, hybrid systems works such as $[24,25]$ that cover delays employ common Lyapunov functionals that are not needed here.

3.2. Stability of the linear time-varying system. The main result of this subsection is a stability result for the linear time-varying system (35); see Remark 3.6 for the potential benefits of our approach as compared to analyzing the time-varying system directly. Our main result in this subsection is the following.

THEOREM 3.5. If the system (35) satisfies Assumptions 5-8, then it is uniformly globally exponentially stable to 0 .

Proof. For each $j \in\{1,2, \ldots, k\}$, we can rewrite (35) as

$$
\begin{aligned}
\dot{x}(t)= & A_{j} x(t)+B_{j} x\left(t-\tau_{j}\right)+\left[A(t)-A_{j}\right] x(t)+\left[B(t)-B_{j}\right] x\left(t-\tau_{j}\right) \\
& +B(t)\left[x(t-\tau(t))-x\left(t-\tau_{j}\right)\right] .
\end{aligned}
$$

Consequently, the system (35) can be represented as this system with switches:

$$
\begin{aligned}
\dot{x}(t)= & A_{\sigma(t)} x(t)+B_{\sigma(t)} x\left(t-\tau_{\sigma(t)}\right)+\Lambda\left(t, \sigma(t), x_{t}\right), \text { where } \\
\left|\Lambda\left(t, j, x_{t}\right)\right| \leq & \left|\left[A(t)-A_{j}\right] x(t)\right|+\left|\left[B(t)-B_{j}\right] x\left(t-\tau_{j}\right)\right| \\
& +|B(t)| \int_{t-\max \left\{\tau_{j}, \tau(t)\right\}}^{t-\min \left\{\tau_{j}, \tau(t)\right\}}|A(\ell) x(\ell)+B(\ell) x(\ell-\tau(\ell))| \mathrm{d} \ell
\end{aligned}
$$

for all $t \in\left[t_{j}, t_{j+1}\right)$ and $j \in \mathbb{Z}_{\geq 0}$, by extending $A$ and $B$ to be constant on $(-\infty, 0]$ and recalling our assumption that throughout this section, the initial functions are also constant on $(-\infty, 0]$. (This assumption was needed in order to apply the fundamental theorem of calculus to get (43) for all values of $t \geq 0$.)

Assumption 6 implies that

$$
\begin{aligned}
\left|\Lambda\left(t, \sigma(t), x_{t}\right)\right| \leq & \bar{a}|x(t)|+\bar{b}\left|x\left(t-\tau_{\sigma(t)}\right)\right| \\
& +b_{b}\left|\int_{t-\tau_{\sigma(t)}}^{t-\tau(t)}\left[a_{b}|x(\ell)|+b_{b}|x(\ell-\tau(\ell))|\right] \mathrm{d} \ell\right| \\
\leq & \bar{a}|x(t)|+\bar{b}\left|x\left(t-\tau_{\sigma(t)}\right)\right| \\
& +b_{b} \bar{\tau}\left(a_{b}+b_{b}\right) \sup _{\ell \in\left[t-2 \tau_{b}-\max _{i} \tau_{i}, t\right]}|x(\ell)| \\
\leq & {\left[\bar{a}+\bar{b}+b_{b} \bar{\tau}\left(a_{b}+b_{b}\right)\right] \sup _{\ell \in\left[t-2 \tau_{b}-\max _{i} \tau_{i}, t\right]}|x(\ell)| . }
\end{aligned}
$$

We deduce from Assumption 7 that

$$
\begin{aligned}
\dot{V}_{\sigma(t)}(t) \leq & \alpha_{\sigma(t)} V_{\sigma(t)}\left(x_{t}\right) \\
& +\frac{\gamma_{\sigma(t)}\left[\bar{a}+\bar{b}+b_{b} \bar{\tau}\left(a_{b}+b_{b}\right)\right]^{2}}{\Psi_{1}} \sup _{\ell \in\left[t-2 \tau_{b}-\max _{i} \tau_{i}, t\right]} \Psi_{1}|x(\ell)|^{2}
\end{aligned}
$$

for almost all $t \geq 0$. Hence, Assumptions 1-4 are satisfied by (43) with $W(x)=\Psi_{1}|x|^{2}$ and $\delta=0$. Then Theorem 2.4 allows us to conclude global uniform asymptotic 
stability to 0 . In fact, the proof of Theorem 2.4 (specialized to the cases where $\delta=0$ ) provides an exponential decay estimate on $U\left(t, x_{t}\right)=V_{\sigma(t)}\left(t, x_{t}\right)$. By using (39), we deduce that (35) is also uniformly globally exponentially stable to 0 . This completes the proof.

Remark 3.6. Our analysis shows that we can replace the assumption that the initial functions are constant on $(-\infty, 0]$ by the assumption that they are constant on $\left[-\max \left\{\tau_{b}, \max _{i} \tau_{i}\right\}, 0\right]$. Some advantages of the approach in this and the next subsection are that we do not require the delays to be $C^{1}$, and that in the special case of $C^{1}$ delays $\tau(t)$, we allow $\dot{\tau}(t) \geq 1$ to hold for some choices of $t$. This has implications for communication network applications, since for example, when packets are delivered over a network and arrive in the wrong order, $t-\tau(t)$ can have an inverse but the bound $\dot{\tau}(t)<1$ on the derivative does not hold and the delay is not continuous in time. Moreover, our delay compensating feedback controls in the next subsection do not contain any distributed terms that usually arise from reduction model controllers.

3.3. State feedback design for linear time-varying systems with input delay. In this subsection, we show how a control law with switchings (and a piecewise constant gain) can be used to stabilize a time-varying system that need not have switchings. Consider the system

$$
\dot{x}(t)=A(t) x(t)+B_{0}(t) u(t-\tau(t))
$$

under the feedback $u(t-\tau(t))=K_{\sigma(t)} x(t-\tau(t)), t \geq 0$, where $x$ takes values in $\mathbb{R}^{n}$ for any $n \in \mathbb{N}, u$ is valued in $\mathbb{R}^{p}$ for any $p \in \mathbb{N}$, and the initial function $\phi \in C_{\text {in }}^{0}$ is constant on $(-\infty, 0]$. Assume the following.

Assumption 9. The functions $A, B_{0}$, and $\tau$ are piecewise $C^{1}$, and there exist constants $a_{b}, b_{b}$, and $\tau_{b}$ such that

$$
|A(t)| \leq a_{b}, \quad\left|B_{0}(t)\right| \leq b_{0, b}, \text { and } \tau(t) \leq \tau_{b}
$$

hold for all $t \geq 0$.

Assumption 10. There exist an integer $k \in \mathbb{N}$, a switching signal $\sigma:[0,+\infty) \rightarrow$ $\{1,2, \ldots, k\}$ whose switching instants $\left\{t_{i}\right\}$ admit positive constants $\mathcal{T}_{1}$ and $\mathcal{T}_{2}$ that satisfy (2) for all $i \in \mathbb{Z}_{\geq 0}$, a finite set $\left\{\left(A_{i}, B_{0, i}, \tau_{i}\right): i \in\{1,2, \ldots, k\}\right\}$ in $\mathbb{R}^{n \times n} \times$ $\mathbb{R}^{n \times n} \times[0,+\infty)$, and nonnegative constants $\bar{a}, \bar{b}_{0}$, and $\bar{\tau}$ such that

$$
\left|A(t)-A_{\sigma(t)}\right| \leq \bar{a}, \quad\left|B_{0}(t)-B_{0, \sigma(t)}\right| \leq \bar{b}_{0}, \quad \text { and } \quad\left|\tau(t)-\tau_{\sigma(t)}\right| \leq \bar{\tau}
$$

hold for all $t \geq 0$.

Assumption 11. For each $i \in\{1, \ldots, k\}$, there exist a locally absolutely continuous function $V_{i}: C_{\text {in }} \rightarrow[0,+\infty)$, a matrix $K_{i}$, a real constant $\alpha_{i}$, and a positive constant $\gamma_{i}$ such that along all trajectories of

$$
\dot{z}(t)=A_{i} z(t)+B_{0, i} K_{i} z\left(t-\tau_{i}\right)+\delta_{i}(t)
$$

for all piecewise continuous functions $\delta_{i}:[0,+\infty) \rightarrow \mathbb{R}^{n}$, the time derivative of $V_{i}$ satisfies

$$
\dot{V}_{i} \leq \alpha_{i} V_{i}\left(z_{t}\right)+\gamma_{i}\left|\delta_{i}(t)\right|^{2}
$$


for almost all $t$, where the triples $\left(A_{i}, B_{0, i}, \tau_{i}\right)$ are from Assumption 10. Moreover, there are constants $\Psi_{1}>0$ and $\Psi_{2}>0$ such that for all $\phi \in C_{\text {in }}$ and all $i \in\{1, \ldots, k\}$, we have

$$
\Psi_{1}|\phi(0)|^{2} \leq V_{i}(\phi) \leq \Psi_{2}|\phi|_{\left[-\tau_{b}, 0\right]}^{2}
$$

Also, the $\alpha_{i}$ 's satisfy Assumption 3 with $\sigma$ as defined in Assumption 10. Finally, there is a constant $\mu>1$ such that $V_{i}(\phi) \leq \mu V_{j}(\phi)$ holds for all $(i, j) \in\{1, \ldots, m\}^{2}$ and for all $\phi \in C_{\mathrm{in}}^{0}$.

Assumption 12. Assumption 4 is satisfied, where $\nu$ is a constant that satisfies (8) as before, and $\sigma, \mu$, and the $\alpha_{i}$ 's are defined as in Assumptions 9-11, and the $\beta_{i}$ 's defined by

$$
\beta_{i}=\frac{\gamma_{i}\left[\bar{a}+\bar{b}+b_{b} \bar{\tau}\left(a_{b}+b_{b}\right)\right]^{2}}{\Psi_{1}}
$$

with $\bar{b}=\bar{b}_{0} \max _{j \in\{1, \ldots, k\}}\left|K_{j}\right|$ and $b_{b}=b_{0, b} \max _{j \in\{1, \ldots, k\}}\left|K_{j}\right|$ for $i=1,2, \ldots, k$.

We can now prove the following.

TheOREm 3.7. If the system (46) satisfies Assumptions 9-12, then (46) in a closed loop with the control law

$$
u(t-\tau(t))=K_{\sigma(t)} x(t-\tau(t))
$$

is uniformly globally exponentially stabilized to 0.

Proof. The closed loop system is

$$
\dot{x}(t)=A(t) x(t)+B(t) x(t-\tau(t))
$$

with $B(t)=B_{0}(t) K_{\sigma(t)}$. Let $B_{i}=B_{0, i} K_{i}$ for $i=1,2, \ldots, k$. Then

$$
\left|B(t)-B_{\sigma(t)}\right|=\left|B_{0}(t) K_{\sigma(t)}-B_{0, \sigma(t)} K_{\sigma(t)}\right| \leq\left|B_{0}(t)-B_{0, \sigma(t)}\right|\left|K_{\sigma(t)}\right| .
$$

From Assumption 10, it follows that

$$
\left|B(t)-B_{\sigma(t)}\right| \leq \bar{b}_{0} \max _{j \in\{1, \ldots, k\}}\left|K_{j}\right| .
$$

Also, using Assumption 9, we can prove that $|B(t)| \leq b_{0, b} \max _{j \in\{1, \ldots, k\}}\left|K_{j}\right|$ for all $t \geq 0$. One can now check that Assumptions 9-12 imply that the system (54) satisfies Assumptions 5-8. Then Theorem 3.5 allows us to conclude.

A key feature that makes the preceding result useful is the design of the feedback gains $K_{i}$, once the sequence $\left\{\left(A_{i}, B_{0, i}, \tau_{i}\right)\right\}$ of triples is fixed.

Remark 3.8. At each time $t \geq 0$, Theorem 3.7 says to utilize the control value $u(t-\tau(t))$ from (53) in the system (46). This control value is computed at each time $t \geq 0$ in terms of (i) the past value of the state vector $x$ at time $t-\tau(t)$ and (ii) the current value $\sigma(t)$ of the switching signal, which is used to determine the choice of the gain matrix $K_{\sigma(t)}$ in (53). Hence, at each time $t \geq 0$, no future knowledge of the switching signal is needed to compute the control.

Copyright $@$ by SIAM. Unauthorized reproduction of this article is prohibited. 
4. Examples. While our work requires several assumptions, we can provide general constructive conditions where the required Lyapunov functions or functionals are being constructed. For instance, if Assumptions 1-4 are satisfied except with the delay bound $\tau_{b}$ replaced by $\tau_{b}=0$ for some functions $V_{i}$ and with $T>\mathcal{T}_{2}$, then we can impose standard growth conditions on the dynamics to satisfy the assumptions for small enough $\tau_{b}>0$. This can be done by transforming each of the $V_{i}$ 's into a Lyapunov-Krasovskii functional. See earlier work [31] where analogous transformations were done in the absence of switching for the special case where the $\alpha_{i}$ 's are negative, but similar reasoning applies in our more general case where the systems have switching and where some of the $\alpha_{i}$ 's can be nonnegative. See also Appendix C below for general Lyapunov constructions. The following examples illustrate how to satisfy our assumptions.

4.1. Periodic system. Consider the switched linear system

$$
\dot{x}(t)=A_{\sigma(t)} x(t)+B x(t-\tau(t))
$$

with $x$ valued in $\mathbb{R}^{2}, \tau$ valued in $\left[0, \tau_{b}\right]$ for any bound $\tau_{b}>0$,

$$
A_{1}=-I, \quad A_{2}=\left[\begin{array}{ll}
-2 & -1 \\
-1 & -2
\end{array}\right], \text { and } B=\left[\begin{array}{ll}
b & b \\
b & b
\end{array}\right]
$$

with $b \geq 0$ being a constant, and $\sigma:[0,+\infty) \rightarrow\{1,2\}$ being periodic of some period $\mathcal{P}>0$ and defined by $\sigma(t)=1$ when $t \in[0, \mathcal{P} / 2)$ and $\sigma(t)=2$ when $t \in[\mathcal{P} / 2, \mathcal{P})$. Assume that $\mathcal{P} \geq 2 \tau_{b} / 3$. We provide conditions (on $b$ and $\mathcal{P}$ ) ensuring that the origin of (57) is uniformly globally asymptotically stable.

We use the positive definite quadratic functions $V_{1}(x)=\sqrt{3}|x|^{2} / 2$ and $V_{2}(x)=$ $x_{1}^{2}+x_{1} x_{2}+x_{2}^{2}$. Then for all $t \in\left[i \mathcal{P}, \frac{\mathcal{P}}{2}+i \mathcal{P}\right)$ and integers $i \in \mathbb{Z}_{\geq 0}$, we have

$$
\dot{V}_{1}(t) \leq-2 V_{1}(x(t))+4 b \sup _{\ell \in\left[t-\tau_{b}, t\right]} V_{1}(x(\ell))
$$

and when $t \in\left[i \mathcal{P}+\frac{\mathcal{P}}{2}, i \mathcal{P}+\mathcal{P}\right)$ and $i \in \mathbb{Z}_{\geq 0}$, we instead have

$$
\dot{V}_{2}(t) \leq-2 V_{2}(x(t))+(4 \sqrt{3}) b \sup _{\ell \in\left[t-\tau_{b}, t\right]} V_{1}(x(\ell)),
$$

along all solutions of (57), where we used the triangle inequality to get the coefficients of $b$ in (59)-(60). Also, $V_{2}(x) \leq \sqrt{3} V_{1}(x)$ and $V_{1}(x) \leq \sqrt{3} V_{2}(x)$ hold for all $x \in \mathbb{R}^{2}$. Then, with our notation from Assumptions 1-2, we can choose $W=V_{1}, \mathcal{T}_{2}=\mathcal{P} / 2$, any constant $\mathcal{T}_{1} \in(0, \mathcal{P} / 2), \mu=\sqrt{3}, \alpha_{1}=-2, \alpha_{2}=-2, \beta_{1}=4 b$, and $\beta_{2}=(4 \sqrt{3}) b$. Also, choosing $T=2 \mathcal{P}$, our assumption that $\mathcal{P} \geq 2 \tau_{b} / 3$ gives $T=\mathcal{P} / 2+3 \mathcal{P} / 2 \geq$ $\mathcal{P} / 2+\tau_{b}=\mathcal{T}_{2}+\tau_{b}$, and we also have

$$
\int_{t-T}^{t} \alpha_{\sigma(\ell)} \mathrm{d} \ell=-2 T=-4 \mathcal{P}
$$

for all $t \geq T$. Thus, Assumption 3 also holds with $\lambda(T)=4 \mathcal{P}$, so Assumptions 1-3 are satisfied. Also, we can choose $\nu=(2 \sqrt{3}) b\left(1-e^{-4 \mathcal{P}}\right)$. Moreover, $L(2 \mathcal{P})=4$.

From (9) and Theorem 2.4, we deduce that if

$$
\begin{aligned}
& \mu^{L(T)+1} e^{-\lambda(T)}+\left[\frac{\mu^{L(T)+2}-1}{\mu-1}-L(T)-1\right] \nu \mu \\
& =9 \sqrt{3} e^{-4 \mathcal{P}}+\left[\frac{26}{\sqrt{3}-1}-5\right] 6 b\left(1-e^{-4 \mathcal{P}}\right)<1
\end{aligned}
$$

Copyright (c) by SIAM. Unauthorized reproduction of this article is prohibited. 
then (57) is uniformly globally asymptotically stable to 0 . Roughly speaking, the system (57) is uniformly globally asymptotically stable to 0 if $\mathcal{P}$ is large enough and $b$ is sufficiently small.

4.2. Switched state feedback design for a nonperiodic system. We apply Theorem 3.7 to the linear system

$$
\dot{x}(t)=A(t) x(t)+B_{0} u(t-\tau(t))
$$

with a time-varying delay $\tau$, where $B_{0}=\left[\begin{array}{ll}0 & 1\end{array}\right]^{\top}, \tau$ is defined on intervals of the form $[i \mathcal{P},(i+1) \mathcal{P})$ for all integers $i \geq 0$ by $(\mathrm{a}) \tau(t)=\tau_{s}$ if $t \in\left[i \mathcal{P},\left(i+q_{i}\right) \mathcal{P}\right)$ and $(\mathrm{b})$ $\tau(t)=\tau_{b}$ if $t \in\left[\left(i+q_{i}\right) \mathcal{P},(i+1) \mathcal{P}\right)$ where $\tau_{s} \in[0,1 / 12]$ and $\tau_{b} \in(1 / 12, \mathcal{P}]$ and $\mathcal{P}>0$ are all given constants and the constants $q_{i}$ admit constants $\underline{q}>0$ and $\bar{q} \in(0,1)$ such that $\underline{q} \leq q_{i} \leq \bar{q}$ for all $i$, and where the function $A:[0,+\infty) \rightarrow \mathbb{R}^{2 \times 2}$ is defined by

$$
\begin{aligned}
A(t) & =\left[\begin{array}{cc}
-1 & 0 \\
0 & 0
\end{array}\right] \text { for all } t \in\left[i \mathcal{P},\left(i+q_{i}\right) \mathcal{P}\right) \text { and } i \geq 0 \\
\text { and } A(t) & =\left[\begin{array}{cc}
1 & 2 \\
-2 & -1
\end{array}\right] \text { for all } t \in\left[\left(i+q_{i}\right) \mathcal{P},(i+1) \mathcal{P}\right) \text { and } i \geq 0
\end{aligned}
$$

Step 1 . We choose the switching times $t_{2 j}=j \mathcal{P}$ and $t_{2 j+1}=q_{j} \mathcal{P}+j \mathcal{P}$ for all $j \geq 0$, and define $\sigma$ by $\sigma(t)=1$ when $t \in\left[t_{2 j}, t_{2 j+1}\right)$ and $\sigma(t)=2$ when $t \in\left[t_{2 j+1}, t_{2(j+1)}\right)$ for all $j \in \mathbb{Z}_{\geq 0}$. Set $A_{\sigma(t)}=A(t)$ for all $t \geq 0$. We select $u(t-\tau(t))=K_{\sigma(t)} x(t-\tau(t))$ with

$$
K_{1}=\left[\begin{array}{ll}
0 & -e^{-\tau_{s}}
\end{array}\right] \text { and } K_{2}=0 .
$$

The eigenvalues of $A_{1}+B_{0} K_{1}$ are $\left\{-1,-e^{-\tau_{s}}\right\}$, and those of $A_{2}+B_{0} K_{2}=A_{2}$ are $\pm \sqrt{3} i$, so the feedback system corresponding to the mode $\sigma(t)=2$ is not asymptotically stable to 0 when the delay is 0 .

Step 2. We study properties of the subsystem

$$
\left\{\begin{array}{l}
\dot{z}_{1}(t)=-z_{1}(t)+\delta_{11}(t) \\
\dot{z}_{2}(t)=-e^{-\tau_{s}} z_{2}\left(t-\tau_{s}\right)+\delta_{12}(t)
\end{array}\right.
$$

for the mode $\sigma(t)=1$, where $\delta_{1}=\left(\delta_{11}, \delta_{12}\right)$; see (49). Set

$$
S\left(z_{2, t}\right)=\int_{t-\tau_{s}}^{t} e^{2(m-t)} z_{2}^{2}(m) \mathrm{d} m+6 \mathcal{Z}^{2}(t),
$$

$$
\text { where } \mathcal{Z}(t)=z_{2}(t)-\int_{t-\tau_{s}}^{t} e^{m-t} z_{2}(m) \mathrm{d} m \text {. }
$$

Along all solutions of (65), we have

$$
\begin{aligned}
\dot{S}(t)= & -2 \int_{t-\tau_{s}}^{t} e^{2(m-t)} z_{2}^{2}(m) \mathrm{d} m+z_{2}^{2}(t)-e^{-2 \tau_{s}} z_{2}^{2}\left(t-\tau_{s}\right) \\
& +12 \mathcal{Z}(t)\left(\delta_{12}(t)-\mathcal{Z}(t)\right) .
\end{aligned}
$$

Copyright $@$ by SIAM. Unauthorized reproduction of this article is prohibited. 
We can use Young's and then Jensen's inequalities to get

$$
\begin{aligned}
z_{2}^{2}(t) & =\left[\mathcal{Z}(t)+\int_{t-\tau_{s}}^{t} e^{m-t} z_{2}(m) \mathrm{d} m\right]^{2} \\
& \leq \frac{3}{2}\left(\int_{t-\tau_{s}}^{t} e^{m-t} z_{2}(m) \mathrm{d} m\right)^{2}+3 \mathcal{Z}^{2}(t) \\
& \leq \frac{3 \tau_{s}}{2} \int_{t-\tau_{s}}^{t} e^{2(m-t)} z_{2}^{2}(m) \mathrm{d} m+3 \mathcal{Z}^{2}(t),
\end{aligned}
$$

since $(a+b)^{2} \leq 1.5 a^{2}+3 b^{2}$ holds for all $a \geq 0$ and $b \geq 0$. Using (68) to upper bound $z_{2}^{2}(t)$ in $(67)$ gives

$$
\dot{S}(t) \leq-\int_{t-\tau_{s}}^{t} e^{2(m-t)} z_{2}^{2}(m) \mathrm{d} m-9 \mathcal{Z}^{2}(t)+12\{\mathcal{Z}(t)\}\left\{\delta_{12}(t)\right\},
$$

since $\tau_{s} \leq 2 / 3$. By applying the triangle inequality $a b \leq 0.5\left(a^{2}+b^{2}\right)$, where $a$ and $b$ are the terms in curly braces in (69), it follows that the time derivative of $V_{1}\left(z_{t}\right)=S\left(z_{2, t}\right)+3 z_{1}^{2}(t)$ along all solutions of (65) satisfies

$$
\begin{aligned}
\dot{V}_{1}(t) & \leq-3 z_{1}^{2}-\int_{t-\tau_{s}}^{t} e^{2(m-t)} z_{2}^{2}(m) \mathrm{d} m+6\left|\delta_{1}(t)\right|^{2}-3 \mathcal{Z}^{2}(t) \\
& \leq-\frac{1}{2} V_{1}\left(z_{t}\right)+6\left|\delta_{1}(t)\right|^{2}
\end{aligned}
$$

since $6 z_{1}(t) \delta_{11}(t) \leq 3 z_{1}^{2}(t)+3 \delta_{11}^{2}(t)$ holds for all $t \geq 0$.

Step 3 . We study properties of the subsystem

$$
\dot{z}_{1}=z_{1}+2 z_{2}+\delta_{21}, \dot{z}_{2}=-2 z_{1}-z_{2}+\delta_{22},
$$

corresponding to the switching mode $\sigma(t)=2$, using the functions $U_{1}(z)=z_{1}^{2}+z_{2}^{2}+$ $z_{1} z_{2}$ and

$$
U_{2}\left(z_{t}\right)=U_{1}(z(t))+\frac{1}{2} \int_{t-\tau_{s}}^{t} z_{2}^{2}(m) \mathrm{d} m
$$

Along all solutions of (71), we have $\dot{U}_{2}(t) \leq \frac{1}{2} z_{2}^{2}(t)+\left\{2 z_{1}(t)+z_{2}(t)\right\}\left\{\delta_{21}(t)\right\}$ $+\left\{2 z_{2}(t)+z_{1}(t)\right\}\left\{\delta_{22}(t)\right\}$. By applying Young's inequality $a b \leq 0.25 a^{2}+b^{2}$ twice (with $a$ and $b$ being the corresponding terms in curly braces), we obtain $\dot{U}_{2}(t) \leq$ $2 U_{2}\left(z_{t}\right)+\left|\delta_{2}(t)\right|^{2}$ along all solutions of $(71)$.

Also, our choice of $V_{1}$ and the Cauchy and Hölder inequalities give

$$
\begin{aligned}
V_{1}\left(z_{t}\right) \leq & \int_{t-\tau_{s}}^{t} e^{2(m-t)} z_{2}^{2}(m) \mathrm{d} m+3 z_{1}^{2}(t) \\
& +12\left[z_{2}^{2}(t)+\left(\int_{t-\tau_{s}}^{t} e^{m-t} z_{2}(m) \mathrm{d} m\right)^{2}\right] \\
\leq & \int_{t-\tau_{s}}^{t} z_{2}^{2}(m) \mathrm{d} m+3 z_{1}^{2}(t)+12 z_{2}^{2}(t) \\
& +12 \int_{t-\tau_{s}}^{t} e^{2(m-t)} \mathrm{d} m \int_{t-\tau_{s}}^{t} z_{2}^{2}(m) \mathrm{d} m \\
\leq & 7 \int_{t-\tau_{s}}^{t} z_{2}^{2}(m) \mathrm{d} m+3 z_{1}^{2}(t)+12 z_{2}^{2}(t) .
\end{aligned}
$$

Copyright (c) by SIAM. Unauthorized reproduction of this article is prohibited. 
Here and in the following, the inequalities and equalities are for all $t \geq 0$. Also, using the fact that the triangle inequality also gives

$$
-2 z_{2}(t) \int_{t-\tau_{s}}^{t} e^{m-t} z_{2}(m) \mathrm{d} m \geq-\frac{13}{14} z_{2}^{2}(t)-\frac{14}{13}\left(\int_{t-\tau_{s}}^{t} e^{m-t} z_{2}(m) \mathrm{d} m\right)^{2},
$$

we can use our bound $\tau_{s} \leq 1 / 12$ to obtain

$$
\begin{aligned}
V_{1}\left(z_{t}\right)= & \int_{t-\tau_{s}}^{t} e^{2(m-t)} z_{2}^{2}(m) \mathrm{d} m+3 z_{1}^{2}(t) \\
& +6\left[z_{2}^{2}(t)+\left\{\int_{t-\tau_{s}}^{t} e^{m-t} z_{2}(m) \mathrm{d} m\right\}^{2}-2 z_{2}(t) \int_{t-\tau_{s}}^{t} e^{m-t} z_{2}(m) \mathrm{d} m\right] \\
\geq & \frac{25}{26} \int_{t-\tau_{s}}^{t} e^{2(m-t)} z_{2}^{2}(m) \mathrm{d} m+3 z_{1}^{2}(t)+\frac{3}{7} z_{2}^{2}(t) \\
\geq & \frac{25 e^{-\frac{1}{6}}}{26} \int_{t-\tau_{s}}^{t} z_{2}^{2}(m) \mathrm{d} m+\frac{3}{7}\left(z_{1}^{2}(t)+z_{2}^{2}(t)\right)
\end{aligned}
$$

by applying Jensen's inequality to the integral in curly braces, after using (74). We deduce that

$$
U_{2}\left(z_{t}\right) \leq \frac{3}{2}\left(z_{1}^{2}+z_{2}^{2}\right)+\frac{1}{2} \int_{t-\tau_{s}}^{t} z_{2}^{2}(m) \mathrm{d} m \leq \frac{7}{2} V_{1}\left(z_{t}\right) .
$$

Also, (73) gives

$$
V_{1}\left(z_{t}\right) \leq 7 \int_{t-\tau_{s}}^{t} z_{2}^{2}(m) \mathrm{d} m+12 z_{1}^{2}(t)+12 z_{2}^{2}(t) \leq 24 U_{2}\left(z_{t}\right)
$$

Choose $V_{2}=2 U_{2}$. Then $V_{1}\left(z_{t}\right) \leq \mu V_{2}\left(z_{t}\right)$ and $V_{2}\left(z_{t}\right) \leq \mu V_{1}\left(z_{t}\right)$ hold for all $t \geq 0$ with the choice $\mu=12$.

Step 4 . We choose $T=2 \mathcal{P}$. With the notation of section 3.3, we can choose $\bar{a}=0, \bar{b}=0, \mathcal{T}_{2}=\max \{\bar{q}, 1-q\} \mathcal{P}, \bar{\tau}=0, \beta_{1}=\beta_{2}=0, \nu=0, \alpha_{1}=-\frac{1}{2}$, and $\alpha_{2}=2$. We have $T=2 \mathcal{P} \geq \overline{\mathcal{P}}+\tau_{b} \geq \mathcal{T}_{2}+\tau_{b}$. We deduce that we can take $\lambda(T)=\frac{1}{2} \underline{q} \mathcal{P}-2(1-\underline{q}) \mathcal{P}=\frac{1}{4} \underline{q} T-(1-\underline{q}) T$. In addition, notice that $L(T)=4$. Then, $V_{1}$ and $V_{2}$ satisfy (39) for suitable constants $\Psi_{1}>0$ and $\Psi_{2}>0$.

From Assumption 4, we deduce from Theorem 3.7 that the following is a sufficient condition for global asymptotic stability: $(12)^{5}<e^{\left[\frac{5}{4} \underline{q}-1\right] T}$, which is equivalent to

$$
\frac{2 \ln (12)}{\mathcal{P}}+\frac{4}{5}<\underline{q}
$$

since $T=2 \mathcal{P}$. The inequality (76) agrees with our intuition that the interval where (62) is unstable should be sufficiently small and the switchings should not be too frequent.

5. Conclusions. We presented a new technique making it possible to establish the ISS of switched time-varying systems with time-varying delays and uncertainties. We used the result for switched controller design for the stability of a class of linear time-varying systems. Our technique used a novel trajectory-based approach. It applies to a wide family of systems for which no other technique of stability analysis was available in the literature. When applied to time-varying linear systems, our result 
only requires the construction of simple Lyapunov-Krasovskii functionals. Control designs under suitable optimality properties will be subjects of future works.

Appendix A. Key lemma and discussion on Halanay inequality. We restate [28, Lemma 1] which we used in our proof of Theorem 2.4. For a proof of this lemma, see [28].

Lemma A1. Let $T^{*}>0$ be a constant. Let a piecewise continuous function $\bar{w}:\left[-T^{*},+\infty\right) \rightarrow[0,+\infty)$ admit a sequence of real numbers $v_{i}$ and positive constants $\bar{v}_{a}$ and $\bar{v}_{b}$ such that $v_{0}=0, v_{i+i}-v_{i} \in\left[\bar{v}_{a}, \bar{v}_{b}\right]$ for all $i \geq 0, \bar{w}$ is continuous on each interval $\left[v_{i}, v_{i+1}\right)$ for all $i \geq 0$, and $\bar{w}\left(v_{i}^{-}\right)$exists and is finite for each $i \in \mathbb{Z}_{\geq 0}$. Let $d:[0,+\infty) \rightarrow[0,+\infty)$ be any piecewise continuous function, and assume that there is a constant $\rho \in(0,1)$ such that $\bar{w}(t) \leq \rho|\bar{w}|_{\left[t-T^{*}, t\right]}+d(t)$ holds for all $t \geq 0$. Then

$$
\bar{w}(t) \leq|\bar{w}|_{\left[-T^{*}, 0\right]} e^{\frac{\ln (\rho)}{T^{*}} t}+\frac{1}{(1-\rho)^{2}}|d|_{[0, t]}
$$

holds for all $t \geq 0$.

The preceding lemma can be viewed as a variant of the following lemma whose conclusion is referred to as Halanay's inequality $[2,15]$.

Lemma A2. Consider a positive valued continuous real valued function $x(t)$ that admits real values $t_{0}, \bar{D}, a$, and $b$ such that $a \geq b \geq 0$ and $\bar{D}>0$ and such that $\dot{x}(t) \leq-a x(t)+b \max \{x(t+r):-\bar{D} \leq r \leq 0\}$ holds for all $t \geq t_{0}$. Then there exists a constant $\gamma \geq 0$ such that $x(t) \leq \max \left\{x\left(t_{0}+r\right):-\bar{D} \leq r \leq 0\right\} e^{-\gamma\left(t-t_{0}\right)}$ holds for all $t \geq t_{0}$.

Lemma A1 can be viewed as a perturbed discrete time version of Lemma A2, since in place of having a decay condition on $x(t)$ in terms of $\dot{x}(t)$ with an overshoot term, we use the constant $\rho \in(0,1)$.

Appendix B. Construction of a special switching signal. When we adopt the technique from section 3 , it is useful to minimize the number of switching instants associated with the function $\sigma$; see Remark 3.2. This motivates the construction of the switching signal $\sigma$ in this appendix. For the sake of simplicity, we consider the case where only $A$ is time varying, but a generalization to the case where $A, B$, and $\tau$ are all time varying is straightforward. Again, for simplicity, $A$ is assumed to be $C^{1}$ on $[0,+\infty)$; we could have taken $A$ to be $C^{1}$ on any finite length interval $\left[T_{1}, T_{2}\right)$ with $T_{1} \geq 0$, and the arguments would be valid to construct a special switching signal on this interval. Then, by concatenating consecutive intervals, we could construct the special switching sequence on $[0,+\infty)$. We prove the following, where choosing $\Delta$ larger reduces the number of switches on any fixed length interval:

Lemma A3. Let $A:[0,+\infty) \rightarrow \mathbb{R}^{n \times n}$ be bounded and $C^{1}$ and admit a constant $d_{a}>0$ such that $\sup _{t \geq 0}|\dot{A}(t)| \leq d_{a}$. Then for each constant $\Delta>0$, we can find a constant $k_{a} \in \mathbb{N}$, constant matrices $A_{i} \in \mathbb{R}^{n \times n}$ for $i=1,2, \ldots, k_{a}$, and a switching signal $\sigma:[0,+\infty) \rightarrow\left\{1,2, \ldots, k_{a}\right\}$ whose switching times $\left\{t_{i}\right\}$ are such that condition (2) holds with $\mathcal{T}_{1}=\Delta / d_{a}$ and $\mathcal{T}_{2}=2 t_{1}$ and such that $\sup _{t \geq 0}\left|A(t)-A_{\sigma(t)}\right| \leq 2 \Delta$.

Proof. Let $a_{b}$ be a constant such that $|A(t)| \leq a_{b}$ for all $t \geq 0$. Let $\Delta>0$ be any constant. The boundedness of $A$ implies the following fact: There are $k_{a} \in \mathbb{N}$ constant matrices $A_{i} \in \mathbb{R}^{n \times n}$ for $i=1, \ldots, k_{a}$ such that for each $t \in[0,+\infty)$, there is an $l \in \mathcal{S}_{k_{a}}$ (depending on $t$ ) with $\mathcal{S}_{k_{a}}=\left\{1, \ldots, k_{a}\right\}$ such that the inequality $\left|A(t)-A_{l}\right|<\Delta$ holds.

For what follows, we can assume that there is no $j \in \mathcal{S}_{k_{a}}$ such that there is an instant $t_{c} \geq 0$ such that $\left|A(t)-A_{j}\right|<2 \Delta$ holds for all $t \geq t_{c}$. Indeed, this case is not 
interesting and trivial (because if such a $j$ existed, then we can choose the switching times $t_{i}$ that we construct in what follows, to be periodic on $\left[t_{c},+\infty\right)$ ). Next, let us define a strictly increasing sequence $\left\{s_{i}\right\}$ of nonnegative real numbers as follows.

(1) Let $s_{0}=0$. The fact above implies that there is $i_{0} \in \mathcal{S}_{k_{a}}$ such that $\left|A\left(s_{0}\right)-A_{i_{0}}\right|<\Delta$.

(2) Let $s_{1}>0$ be such that, for all $t \in\left[s_{0}, s_{1}\right),\left|A(t)-A_{i_{0}}\right|<2 \Delta$ and $\left|A\left(s_{1}\right)-A_{i_{0}}\right|=2 \Delta$. Then, the fact above implies that there is $i_{1} \in \mathcal{S}_{k_{a}}$ such that $\left|A\left(s_{1}\right)-A_{i_{1}}\right|<\Delta$.

(3) We proceed by induction. Induction assumption: we assume we have $s_{0}$, $s_{1}, \ldots, s_{l}$, and $l>0$ with $0=s_{0}<s_{1}<\cdots<s_{l}$ and $i_{0} \in \mathcal{S}_{k_{a}}, \ldots, i_{l} \in$ $\mathcal{S}_{k_{a}}$ such that for all $m \in\{1, \ldots, l\}$, we have $\left|A\left(s_{m}\right)-A_{i_{m-1}}\right|=2 \Delta$ and $\left|A\left(s_{m}\right)-A_{i_{m}}\right|<\Delta$; and for all $t \in\left[s_{m-1}, s_{m}\right)$, we have $\left|A(t)-A_{i_{m-1}}\right|<2 \Delta$.

(4) According to our induction assumption, $\left|A\left(s_{l}\right)-A_{i_{l}}\right|<\Delta$. Therefore there is $s_{l+1}>s_{l}$ such that, for all $t \in\left[s_{l}, s_{l+1}\right),\left|A(t)-A_{i_{l}}\right|<2 \Delta$ and $\left|A\left(s_{l+1}\right)-A_{i_{l}}\right|=2 \Delta$. Let $i_{l+1} \in \mathcal{S}_{k_{a}}$ be such that $\left|A\left(s_{l+1}\right)-A_{i_{l+1}}\right|<\Delta$. The induction assumption is satisfied at the step $i+1$.

Let $\sigma$ be defined by $\sigma(t)=i_{m}$ when $t \in\left[s_{m}, s_{m+1}\right)$. Then for all $t \geq 0$, the inequality $\left|A(t)-A_{\sigma(t)}\right| \leq 2 \Delta$ is satisfied. We next show that $\mathcal{T}_{1} \leq s_{i+1}-s_{i}$ for all $i \in \mathbb{N}$; later we transform the sequence $\left\{s_{i}\right\}$ into a new sequence $\left\{t_{j}\right\}$ that satisfies all of our requirements. For all $m \in \mathbb{N}$, we can use the triangle inequality to obtain

$$
\begin{aligned}
\left|A\left(s_{m-1}\right)-A\left(s_{m}\right)\right| & =\left|A\left(s_{m-1}\right)-A_{i_{m-1}}+A_{i_{m-1}}-A\left(s_{m}\right)\right| \\
& \geq-\left|A\left(s_{m-1}\right)-A_{i_{m-1}}\right|+\left|A_{i_{m-1}}-A\left(s_{m}\right)\right| .
\end{aligned}
$$

Also, for all $q \in \mathbb{N}$, we have $\left|A\left(s_{q}\right)-A_{i_{q-1}}\right|=2 \Delta$ and $\left|A\left(s_{q}\right)-A_{i_{q}}\right|<\Delta$. We deduce that

$$
\begin{aligned}
-\left|A\left(s_{m-1}\right)-A_{i_{m-1}}\right|+\left|A_{i_{m-1}}-A\left(s_{m}\right)\right| & =2 \Delta-\left|A\left(s_{m-1}\right)-A_{i_{m-1}}\right| \\
& \geq 2 \Delta-\Delta .
\end{aligned}
$$

Combining (78) and (79), and recalling our choice of $d_{a}$, we obtain $d_{a}\left(s_{m}-s_{m-1}\right) \geq$ $\left|A\left(s_{m-1}\right)-A\left(s_{m}\right)\right| \geq \Delta$. Therefore, $\Delta \leq d_{a}\left(s_{m}-s_{m-1}\right)$ for all $m \in \mathbb{N}$.

If there is no constant $\mathcal{T}_{2}>0$ such that $s_{i+1}-s_{i} \leq \mathcal{T}_{2}$ for all $i \in \mathbb{N}$, then we can replace the sequence $\left\{s_{i}\right\}$ that we constructed above by a new sequence $\left\{t_{j}\right\}$ which is composed of the $s_{i}$ 's and additional switching times that are introduced to get a sufficiently large constant $\mathcal{T}_{2}>0$ such that $\mathcal{T}_{1} \leq t_{i+1}-t_{i} \leq \mathcal{T}_{2}$ holds for all $i \in \mathbb{Z}_{\geq 0}$. We can construct the $t_{i}$ 's as follows. We choose $t_{0}=0$ and $t_{1}=s_{1}$, and we set $\mathcal{L}_{i}=\operatorname{Floor}\left(\left(s_{i}-s_{i-1}\right) / s_{1}\right)$ for all integers $i \geq 2$. If $\mathcal{L}_{2} \in\{0,1\}$, then we choose $t_{2}=s_{2}$, and so we do not introduce any new switching instants on $\left[0, s_{2}\right]$. On the other hand, if $\mathcal{L}_{2} \geq 2$, then we introduce the switching times $t_{j+1}=s_{1}+j s_{1}$ for $j=1,2, \ldots, \mathcal{L}_{2}-1$, and we set $t_{\mathcal{L}_{2}+1}=s_{2}$. By the definition of the floor function, we have $t_{j} \in\left(s_{1}, s_{2}\right)$ for all $j \in\left\{2,3, \ldots, \mathcal{L}_{2}\right\}$ and $s_{2}-\mathcal{L}_{2} s_{1} \in\left[s_{1}, 2 s_{1}\right]$, so all of the switching times $t_{i}$ on $\left[0, s_{2}\right]$ will satisfy $\mathcal{T}_{1} \leq t_{i+1}-t_{i} \leq 2 s_{1}$. We continue in the same way, by introducing the switching times $s_{p-1}+j s_{1}$ for $j=1,2, \ldots, \mathcal{L}_{p}-1$ on the interval $\left(s_{p-1}, s_{p}\right)$ if $p \geq 2$ is such that $\mathcal{L}_{p} \geq 2$, but not introducing any switching times in the interval $\left(s_{p-1}, s_{p}\right)$ for integers $p \geq 2$ for which $\mathcal{L}_{p} \in\{0,1\}$. Since $s_{1} \geq \mathcal{T}_{1}$, this produces a sequence $t_{i}$ such that $\mathcal{T}_{1} \leq t_{i}-t_{i-1} \leq 2 s_{1}$ for all integers $i \geq 1$ and completes the proof.

Appendix C. Technical result. One can construct LKFs such that (38) and (39) are satisfied. We illustrate the key steps in one such construction using an 
example involving two systems, but this can be directly extended to any arbitrary number of systems. In what follows, $\eta_{7}$ plays the role of the constant $\mu$ appearing in Assumption 2.

Lemma A4. Consider the systems

$$
\dot{X}(t)=A_{a} X(t)+A_{b} X\left(t-T_{a}\right) \text { and } \dot{Z}(t)=B_{a} Z(t)+B_{b} Z\left(t-T_{b}\right),
$$

where $X$ and $Z$ are valued in $\mathbb{R}^{n}$ for any dimension $n$, and $A_{a}, A_{b}, B_{a}$, and $B_{b}$ are constant matrices, and where $T_{b}$ and $T_{a}$ are any nonnegative values. Assume that the systems admit LKFs of the form

$$
\begin{gathered}
Q_{a}\left(X_{t}\right)=X(t)^{\top} P_{a} X(t)+\int_{t-T_{a}}^{t} \theta_{a}(t-\ell) X(\ell)^{\top} R_{a} X(\ell) \mathrm{d} \ell \text { and } \\
Q_{b}\left(Z_{t}\right)=Z(t)^{\top} P_{b} Z(t)+\int_{t-T_{b}}^{t} \theta_{b}(t-\ell) Z(\ell)^{\top} R_{b} Z(\ell) \mathrm{d} \ell,
\end{gathered}
$$

respectively, where $P_{a} \in \mathbb{R}^{n \times n}, P_{b} \in \mathbb{R}^{n \times n}, R_{a} \in \mathbb{R}^{n \times n}$, and $R_{b} \in \mathbb{R}^{n \times n}$ are symmetric positive definite matrices, and where $\theta_{a}$ and $\theta_{b}$ are bounded, continuous, and nonnegative valued functions, such that their time derivatives along the corresponding systems in (80) satisfy

$$
\dot{Q}_{a}(t) \leq-|X(t)|^{2} \text { and } \dot{Q}_{b}(t) \leq-|Z(t)|^{2},
$$

respectively. Then the functionals

$$
\begin{aligned}
Q_{a}^{*}\left(X_{t}\right) & =Q_{a}\left(X_{t}\right)+\frac{1}{2} \int_{t-T_{b}}^{t} e^{-t+\ell}|X(\ell)|^{2} \mathrm{~d} \ell \text { and } \\
Q_{b}^{*}\left(Z_{t}\right) & =Q_{a}\left(Z_{t}\right)+\frac{1}{2} \int_{t-T_{b}}^{t} e^{-t+\ell}|Z(\ell)|^{2} \mathrm{~d} \ell
\end{aligned}
$$

admit positive constants $\eta_{i}>0$ for $i=1,2, \ldots, 7$ such that

$$
\dot{Q}_{a}^{*}(t) \leq-\eta_{1} Q_{a}^{*}\left(X_{t}\right) \text { and } \dot{Q}_{b}^{*}(t) \leq-\eta_{2} Q_{b}^{*}\left(Z_{t}\right)
$$

hold along all solutions of the corresponding systems, and such that

$$
\begin{aligned}
& \eta_{3}|\phi(0)|^{2} \leq Q_{a}^{*}(\phi) \leq \eta_{4} \sup _{\ell \in\left[-T_{b}, 0\right]}|\phi(\ell)|^{2}, \\
& \eta_{5}|\phi(0)|^{2} \leq Q_{b}^{*}(\phi) \leq \eta_{6} \sup _{\ell \in\left[-T_{b}, 0\right]}|\phi(\ell)|^{2},
\end{aligned}
$$

and

$$
Q_{a}^{*}(\phi) \leq \eta_{7} Q_{b}^{*}(\phi) \text { and } Q_{b}^{*}(\phi) \leq \eta_{7} Q_{a}^{*}(\phi)
$$

hold for all $\phi \in C_{\text {in }}$.

Proof. The inequalities (85) are consequences of the definitions of $Q_{a}$ and $Q_{b}$. Simple calculations give

$$
\begin{aligned}
& \dot{Q}_{a}^{*}(t) \leq-\frac{1}{2}|X(t)|^{2}-\frac{1}{2} \int_{t-T_{b}}^{t} e^{-t+\ell}|X(\ell)|^{2} \mathrm{~d} \ell \text { and } \\
& \dot{Q}_{b}^{*}(t) \leq-\frac{1}{2}|Z(t)|^{2}-\frac{1}{2} \int_{t-T_{b}}^{t} e^{-t+\ell}|Z(\ell)|^{2} \mathrm{~d} \ell
\end{aligned}
$$

We easily deduce that (84) and (86) can be satisfied. 
Appendix D. Switching signals that satisfy Assumption 6. Let $f$ : $[0,+\infty) \rightarrow[\underline{f}, \bar{f})$ and $g:[0,+\infty) \rightarrow[\underline{g}, \bar{g})$ be globally Lipschitz functions having $c_{f}$ and $c_{g}$ as positive global Lipschitz constants, respectively. Let $\epsilon>0$ be a real number. We present a systematic way to find vectors $V_{i} \in \mathbb{R}^{2}$ and a switching function $\sigma$ so that $\mathcal{F}(t)=(f(t), g(t))^{\top}$ satisfies $\left|\mathcal{F}(t)-V_{\sigma(t)}\right| \leq \epsilon$ for all $t \geq 0$.

First step. Let $s \in \mathbb{N}$ and let $\nu>0$ be values to be selected later. For each $k \in\{1, \ldots, s\}$, we set $f_{k}=\underline{f}+k(\bar{f}-\underline{f}) / s$ and $g_{k}=\underline{g}+k(\bar{g}-\underline{g}) / s$. Consider the set $\mathcal{E}=\left\{\left(f_{k}, g_{l}\right)^{\top}: k \in\{0, \ldots, s-1\}, l \bar{\in}\{0, \ldots, s-1\}\right\}$ of $s^{2}$ elements in $\mathcal{E}$, which we write as $\mathcal{E}=\left\{V_{1}, \ldots, V_{s^{2}}\right\}$. We define the sequence $t_{i}=i \nu$ for all $i \in \mathbb{Z}_{\geq 0}$.

Second step. For all $i \geq 0$, there exists a pair $(a, b) \in\{0, \ldots, s-1\} \times\{0, \ldots, s-1\}$ such that $\left(f\left(t_{i}\right), g\left(t_{i}\right)\right) \in\left[f_{a}, f_{a+1}\right) \times\left[g_{b}, g_{b+1}\right)$ and if $(c, d) \in\{0, \ldots, s-1\} \times$ $\{0, \ldots, s-1\}$ is different from $(a, b)$, then $\left(f\left(t_{i}\right), g\left(t_{i}\right)\right) \notin\left[f_{c}, f_{c+1}\right) \times\left[f_{d}, f_{d+1}\right)$. We now define $\sigma:[0,+\infty) \rightarrow[0,+\infty)$ as follows: (i) For all $t \in\left[t_{i}, t_{i+1}\right)$, we set $\sigma(t)=\sigma\left(t_{i}\right)$. (ii) Choose $\sigma\left(t_{i}\right)$ such that the choice $V_{\sigma\left(t_{i}\right)}=\left(f_{a}, g_{b}\right)^{\top}$, where $(a, b) \in\{0, \ldots, s-1\} \times\{0, \ldots, s-1\}$, is such that

$$
\left(f\left(t_{i}\right), g\left(t_{i}\right)\right) \in\left[f_{a}, f_{a+1}\right) \times\left[g_{b}, g_{b+1}\right) .
$$

Third step. Let $t \in[0,+\infty)$. Then there is $i \in \mathbb{N}$ such that $t \in\left[t_{i}, t_{i+1}\right)$. Then, from the definition of $\sigma$, it follows that $\mathcal{F}(t)-V_{\sigma(t)}=\mathcal{F}(t)-V_{\sigma\left(t_{i}\right)}=\mathcal{F}\left(t_{i}\right)-V_{\sigma\left(t_{i}\right)}+$ $\mathcal{F}(t)-\mathcal{F}\left(t_{i}\right)$. Consequently, (88) gives

$$
\begin{aligned}
\left|\mathcal{F}(t)-V_{\sigma(t)}\right| & \leq\left|\mathcal{F}\left(t_{i}\right)-V_{\sigma\left(t_{i}\right)}\right|+\left|\mathcal{F}(t)-\mathcal{F}\left(t_{i}\right)\right| \\
& \leq \frac{1}{s} \sqrt{(\bar{f}-\underline{f})^{2}+(\bar{g}-\underline{g})^{2}}+\left|\mathcal{F}(t)-\mathcal{F}\left(t_{i}\right)\right| .
\end{aligned}
$$

Since $f$ and $g$ have the global Lipschitz constants $c_{f}$ and $c_{g}$, respectively, we deduce that

$$
\begin{aligned}
\left|\mathcal{F}(t)-V_{\sigma(t)}\right| & \leq \frac{1}{s} \sqrt{(\bar{f}-\underline{f})^{2}+(\bar{g}-\underline{g})^{2}}+\left(t-t_{i}\right) \sqrt{c_{f}^{2}+c_{g}^{2}} \\
& \leq \frac{1}{s} \sqrt{(\bar{f}-\underline{f})^{2}+(\bar{g}-\underline{g})^{2}}+\nu \sqrt{c_{f}^{2}+c_{g}^{2}} .
\end{aligned}
$$

Fourth step. Choosing $s$ and $\nu$ such that $\frac{2}{\epsilon}|(\bar{f}-\underline{f}, \bar{g}-\underline{g})| \leq s$ and $\nu \leq \epsilon /\left(2\left|\left(c_{f}, c_{g}\right)\right|\right)$ then gives

$$
\frac{1}{s} \sqrt{(\bar{f}-\underline{f})^{2}+(\bar{g}-\underline{g})^{2}}+\nu \sqrt{c_{f}^{2}+c_{g}^{2}} \leq \epsilon .
$$

Thus $\left|\mathcal{F}(t)-V_{\sigma(t)}\right| \leq \epsilon$ for all $t \geq 0$. We can extend this result to matrices of any dimension, by applying analogous arguments to each matrix entry, which provides switching signals that satisfy Assumption 6.

\section{REFERENCES}

[1] N. Bekiaris-Liberis and M. Krstic, Nonlinear Control Under Nonconstant Delays, Adv. Des. Control 25, SIAM, Philadelphia, 2013.

[2] D. Bresch-Pietri, J. Chauvin, and N. Petit, Invoking Halanay inequality to conclude on closed-loop stability of a process with input-varying delay, IFAC Proc., 45 (2012), pp. 266271.

[3] C. BRIAT, Convex conditions for robust stability analysis and stabilization of linear aperiodic impulsive and sampled-data systems under dwell-time constraints, Automatica J. IFAC, 49 (2013), pp. 3449-3457. 
[4] C. BRIAT, Convex conditions for robust stabilization of uncertain switched systems with guaranteed minimum and mode-dependent dwell-time, Systems Control Lett., 78 (2015), pp. 6372.

[5] C. Briat And A. Seuret, Affine minimal and mode-dependent dwell-time characterization for uncertain switched linear systems, IEEE Trans. Automat. Control, 58 (2013), pp. 13041310.

[6] S. Caliskan, H. Özbay, and S-I. Niculescu, Dwell-time computation for stability of switched systems with time delays, IET Control Theory Appl., 7 (2013), pp. 1422-1428.

[7] D. Cheng, J. Wang, And X. Hu, An extension of LaSalle's invariance principle and its application to multi-agent consensus, IEEE Trans. Automat. Control, 53 (2008), pp. 17651770.

[8] G. Chesi, P. Colaneri, J. Geromel, R. Middleton, and R. Shorten, A nonconservative LMI condition for stability of switched systems with guaranteed dwell-time, IEEE Trans. Automat. Control, 57 (2012), pp. 1297-1302.

[9] B. Demirel, C. Briat, And M. Johansson, Supervisory control design for networked systems with time-varying communication delays, Nonlinear Anal. Hybrid Syst., 10 (2013), pp. 94110.

[10] E. FRIDMAn, On robust stability of linear neutral systems with time-varying delays, IMA J. Math. Control Inform., 25 (2008), pp. 393-407.

[11] E. Fridman, Introduction to Time-Delay Systems: Analysis and Control, Birkhäuser, Boston, 2014.

[12] J. C. Geromel and P. Colaneri, Stability and stabilization of continuous-time switched linear systems, SIAM J. Control Optim., 45 (2006), pp. 1915-1930.

[13] R. Goebel, R. Sanfelice, and A. Teel, Hybrid Dynamical Systems. Modeling, Stability, and Robustness, Princeton University Press, Princeton, NJ, 2012.

[14] K. Gu, V. Kharitonov, and J. Chen, Stability of Time-Delay Systems, Birkhäuser, Boston, 2003.

[15] A. Halanay, Differential Equations, Stability, Oscillations, Time Lags, Academic Press, New York, 1966.

[16] J. Hespanha, Uniform stability of switched linear systems: Extensions of La Salle's invariance principle, IEEE Trans. Automat. Control, 49 (2004), pp. 470-482.

[17] J. Hespanha, D. Liberzon, and A. Teel, Lyapunov conditions for input-to-state stability of impulsive systems, Automatica J. IFAC, 44 (2008), pp. 2735-2744.

[18] J. Hespanha And A. Morse, Stability of switched systems with average dwell-time, in Proceedings of the IEEE Conference on Decision and Control, Phoenix, AZ, IEEE, Piscataway, NJ, 1999, pp. 2655-2660.

[19] W. Jiang, E. Fridman, A. Kruszewski, ANd J. Richard, Switching controller for stabilization of linear systems with switched time-varying delays, in Proceedings of the IEEE Conference on Decision and Control, Shanghai, China, 2009, IEEE, Piscataway, NJ, pp. 7923-7928.

[20] H. Khalil, Nonlinear Systems, 3rd ed., Prentice-Hall, Englewood Cliffs, NJ, 2002.

[21] A. Koru, A. Delibasi, And H. ÖzBAy, On dwell time minimization for switched delay systems: Free-weighting matrices method, in Proceedings of the 53rd IEEE Conference on Decision and Control, Los Angeles, CA, IEEE, Piscataway, NJ, 2014, pp. 1978-1982.

[22] T-C. LEE AND Z-P. JIANG, Uniform asymptotic stability of nonlinear switched systems with an application to mobile robots, IEEE Trans. Automat. Control, 53 (2008), pp. 1235-1252.

[23] D. Liberzon, Switching in Systems and Control, Birkhäuser, Berlin, 2003.

[24] J. Liu And A. Teel, Hybrid dynamical systems with finite memory, in Recent Results on Nonlinear Delay Control Systems, I. Karafyllis, M. Malisoff, F. Mazenc, and P. Pepe, eds., Adv. Delays Dyn. 4, Springer, New York, 2016, pp. 261-273.

[25] J. Liu And A. TeEL, Invariance principles for hybrid systems with memory, Nonlinear Anal. Hybrid Syst., 21 (2016), pp. 130-138.

[26] J. Mancilla-Aguilar and R. Garcia, On converse Lyapunov theorems for ISS and iISS switched nonlinear systems, Systems Control Lett., 42 (2001), pp. 47-53.

[27] J. Mancilla-Aguilar and R. Garcia, An extension of LaSalle's invariance principle for switched systems, Systems Control Lett., 55 (2006), pp. 376-384.

[28] F. Mazenc And M. Malisoff, Trajectory based approach for the stability analysis of nonlinear systems with time delays, IEEE Trans. Automat. Control, 60 (2015), pp. 1716-1721.

[29] F. Mazenc And M. Malisoff, Reduction model approach for systems with a time-varying delay, in Proceedings of the 54th IEEE Conference on Decision and Control, Osaka, Japan, IEEE, Piscataway, NJ, 2015, pp. 7723-7727.

[30] F. Mazenc and M. Malisoff, Extension of Razumikhin's theorem for time-varying systems with delay, in Proceedings of the 2016 American Control Conference, Boston, MA, IEEE, Piscataway, NJ, 2016, pp. 84-88.

Copyright (c) by SIAM. Unauthorized reproduction of this article is prohibited. 
[31] F. Mazenc, M. Malisoff, And Z. Lin, Further results on input-to-state stability for nonlinear systems with delayed feedbacks, Automatica J. IFAC, 44 (2008), pp. 2415-2421.

[32] F. Mazenc, M. Malisoff, And S-I. Niculescu, Stability analysis for systems with timevarying delay: Trajectory based approach, in Proceedings of the 54th IEEE Conference on Decision and Control, Osaka, Japan, IEEE, Piscataway, NJ, 2015, pp. 1811-1816.

[33] F. Mazenc, M. Malisoff, And H. ÖZBAy, Stability analysis of switched systems with timevarying discontinuous delays, in Proceedings of the American Control Conference, Seattle, WA, IEEE, Piscataway, NJ, 2017, pp. 5177-5181.

[34] W. Michiels and E. Verriest, A Look at Fast Varying and State Dependent Delays From a System Theory Point of View, Internal Report TW586, University of Leuven, Belgium, 2011, http://www.cs.kuleuven.be/publicaties/rapporten/tw/TW586.abs.html.

[35] A. Monse, Supervisory control of families of linear set-point controllers - Part 1: Exact matching, IEEE Trans. Automat. Control, 41 (1996), pp. 1413-1431.

[36] P. Naghshtabrizi, J. Hespanha, And A. Teel, Exponential stability of impulsive systems with application to uncertain sampled-data systems, Systems Control Lett., 57 (2008), pp. 378385.

[37] P. PEPE, Stabilization in the sample-and-hold sense of nonlinear retarded systems, SIAM J. Control Optim., 52 (2014), pp. 3053-3077.

[38] E. Sontag, Mathematical Control Theory 2nd ed., Springer, New York, 1998.

[39] Z. Sun and S. Ge, Stability Theory of Switched Dynamical Systems, Comm. Control Engrg. Ser., Springer, New York, 2011.

[40] L. Vu and K. Morgansen, Stability of time-delay feedback switched linear systems, IEEE Trans. Automat. Control, 55 (2010), pp. 2385-2390.

[41] Y-E. Wang, X-L. Sun, And F. Mazenc, Stability of switched nonlinear systems with delay and disturbance, Automatica J. IFAC, 69 (2016), pp. 78-86.

[42] F. WiRTh, A converse Lyapunov theorem for linear parameter-varying and linear switching systems, SIAM J. Control Optim., 44 (2005), pp. 210-239.

[43] M. Wu, Y. He, J-H. She, And G-P. Liu, Delay-dependent criteria for robust stability of time-varying delay systems, Automatica J. IFAC, 40 (2004), pp. 1435-1439.

[44] D. XIE AND Y. WU, Stabilisation of time-delay switched systems with constrained switching signals and its applications in networked control systems, IET Control Theory Appl., 4 (2010), pp. 2120-2128.

[45] G. Xie and L. Wang, Periodical stabilization of switched linear systems, J. Comput. Appl. Math., 181 (2005), pp. 176-187.

[46] P. YAN AND H. ÖZBAY, Stability analysis of switched time-delay systems, SIAM J. Control Optim., 47 (2008), pp. 936-949.

[47] P. YAN, H. ÖZBAY, AND M. ŞANSAL, Robust stabilization of parameter varying time delay systems by switched controllers, Appl. Comput. Math., 13 (2014), pp. 31-45.

[48] H. YANG, V. COCQUemPot, AND B. JiAnG, On stabilization of switched nonlinear systems with unstable modes, Systems Control Lett., 58 (2009), pp. 703-708.

[49] B. Zhou AND A. EgOrov, Razumikhin and Krasovskii stability theorems for time-varying delay systems, Automatica J. IFAC, 71 (2016), pp. 281-291.

Copyright (c) by SIAM. Unauthorized reproduction of this article is prohibited. 